\title{
Ecological effects on underdominance threshold drives for vector control
}

\author{
Doran Khamis $^{\mathrm{a}}$, Claire El Mouden ${ }^{\mathrm{a}}$, Klodeta Kura ${ }^{\mathrm{a}}$, Michael B. Bonsall ${ }^{\mathrm{a}, *}$ \\ ${ }^{a}$ Mathematical Ecology Research Group, Department of Zoology, University of Oxford, South Parks Road, Oxford OX1 \\ 3PS, UK
}

\begin{abstract}
Underdominance gene drives are frequency-dependent drives that aim to spread a desired homozygote genotype within a population. When the desired homozygote is released above a threshold frequency, heterozygote fitness disadvantage acts to drive the desired trait to fixation. Underdominance drives have been proposed as a way to control vector-borne disease through population suppression and replacement in a spatially contained and reversible way-benefits that directly address potential safety concerns with gene drives. Here, ecological and epidemiological dynamics are coupled to a model of mosquito genetics to investigate theoretically the impact of different types of underdominance gene drive on disease prevalence. We model systems with two engineered alleles carried either on the same pair of chromosomes at the same locus or homozygously on different pairs at different loci, genetic lethality that affects both sexes or only females, and bi-sex or male-only releases. Further, the different genetic and ecological fitness costs that can arise from genetic modification and artificial rearing are investigated through their effect on the population threshold frequency that is required to trigger the drive mechanism. We show that maleonly releases must be significantly larger than bi-sex releases to trigger the underdominance drive. In addition, we find that female-specific lethality averts a higher percentage of disease cases over a control period than does bi-sex lethality. Decreases in the genetic fitness of the engineered homozygotes can increase the underdominance threshold substantially, but we find that the mating success of transgenic mosquitoes with wild-type females (influenced by a lack of competitiveness or the evolution of behavioural resistance in the form of active female mate preference) and the longevity of artificially-reared mosquitoes are vitally important to the success chances of underdominance based gene drive control efforts.
\end{abstract}

Keywords: gene drive, population genetics, mosquito ecology, epidemiology, mosquito control

\section{Introduction}

2 Vector-borne diseases inflict significant levels of human morbidity and mortality, accounting for $17 \%$

3 of the global disease burden. More than half of the world's population are at risk of contracting a vector-

4 borne disease; more than 300M people contract dengue annually [1], and around 200M malaria infections

5 are reported every year, leading to an estimated 429000 deaths [2]. Genetic vector control technologies

6 may become the first line of defence against diseases for which no vaccine or cure is available, or in

\footnotetext{
* Corresponding author

Email address: Michael.Bonsall@zoo.ox.ac.uk (Michael B. Bonsall)
} 
cases where pathogens have developed resistance to medication. However, there are significant concerns about the safety and viability of these technologies. Thus, the World Health Organisation's Global Vector Response 2017-2030 highlights the need for evidence of the possible impact of new vector interventions [3].

Underdominance refers to traits where heterozygotes are less fit than homozygotes [4]. Consequently, underdominance in a single population is characterised by an unstable equilibrium (the threshold) that acts as a bi-stable switch between the fixation of one allele (in its homozygote genotype) or another. This phenomena may be an economically viable way to ensure an introduced gene reaches fixation if the allele attains a higher frequency than the unstable equilibrium point (through mass-releases of transgenic mosquitoes, for example). This would occur without the need for further investment, through the action of the heterozygote disadvantage. This threshold phenomenon also has two key positive attributes: first, it allows population control efforts to be spatially containable. Migrants from a modified population that enter a wild-type population will be rare (below the threshold) hence the heterozygote disadvantage will drive them to extinction [5-7]. Second, control interventions are naturally reversible: releasing wild-types to a frequency above the threshold will drive them to fixation, thus eradicating the modified population [8]. By contrast, other gene drive-type technologies may be very difficult to contain and are only reversible by releases of further-modified rescue genotypes (although low-fitness varieties of Medea and Wolbachia should be reversible by large-scale wild-type releases [8]). Given these benefits, amid the potential safety concerns over the use of gene drive systems $[9,10]$ and the lack of specific and sufficient regulation [11], underdominance drives are a promising candidate as the self-sustaining genetic control technology of choice for insect pests and disease vectors.

Fully understanding both genetic and ecological fitness costs that modified insects may suffer is vital if underdominance is to be utilised successfully for vector population control. Ecological fitness is affected by differences in mating success, larval development and survival between transgenic and wild strains. Genetic fitness is a combination of the cost intended to cause heterozygote disadvantage and the unintended ambient cost due to genetic modification. Implementations of underdominance in the laboratory and field have had mixed results. Insects with underdominant traits have been created in the laboratory using translocations [12], and studies have investigated their use for the control of mosquitoes [13-16] and other insect pests [17-19]. Usually, population replacement rather than suppression is the goal of the control effort, but translocations [20-22] and other chromosomal rearrangements [23] have also been suggested as methods of population suppression. Translocation-bearing insects typically have low fitness compared to wild counterparts [16, 18], and there has been no successful method of linking a payload gene (e.g. a gene causing refractoriness to disease) to a translocation break point [24]— these issues have meant work has moved away from translocations. Underdominant gene drives have more recently been theorised [25] and realised using engineered alleles with versions of a toxin-antidote system (through maternal toxins targeting genes vital for embryonic development [26] and through RNA interference of a haploinsufficient endogenous gene [5]; approaches collectively known as engineered underdominance), which may assuage the high fitness costs that translocation-bearing insects suffer. 
Our starting point is the engineered underdominance model developed by Davis et al. [25]. Unlike previous extensions of this work [6, 27-29], we avoid simplifying assumptions of hermaphroditic, large (or infinite) populations, where heterozygotes are perfectly non-viable. Instead, we use a continuoustime differential equation ecological model coupled to a population genetics model that governs the offspring proportions. We will account for gender imbalances due to male-only releases and female-specific lethality, imperfect lethality in heterozygotes and genetic fitness costs on the engineered homozygotes. We will also examine the effects of wild-type female mate preference and perturbations to life history parameters of insects carrying engineered alleles. Though the use of underdominance to control disease vector populations has been suggested before, no theoretical study has been undertaken explicitly linking a genetic underdominance model with a mathematical model of a vector-borne disease. We employ an epidemiological model and use it to judge the effects of releasing insects with engineered underdominant alleles.

\section{Models}

A general engineered underdominance (UD) system consists of two mutually dependent alleles, $\alpha$ and $\beta$. Each allele contains a promoter gene that expresses a (partially) lethal toxin, and each also suppresses the toxin expression of the other allele. In this way, heterozygotes of either of the alleles with a wild-type allele suffer a large genetic load, with relative fitness $\phi_{\text {het }}<\phi_{\text {hom }} \leq 1$, where $\phi_{\text {hom }}$ is the fitness of the engineered homozygote (made up of $\alpha$ and $\beta$ in equal proportions). The wild-type genotype has a relative genetic fitness of unity. The fitness costs of the engineered alleles are assumed to act multiplicatively where they are not suppressed.

We consider two UD systems, as proposed by Davis et al. [25]. First, a homologous system, in which $\alpha$ and $\beta$ are carried on the same pair of chromosomes at the same locus; in this case the wild and engineered homozygote genotypes are $A A$ and $\alpha \beta$, respectively. Second, a non-homologous UD system, in which $\alpha$ and $\beta$ are carried homozygously on different pairs of chromosomes at different loci; the wild and engineered homozygote genotypes are $A A B B$ and $\alpha \alpha \beta \beta$, respectively. The non-homologous system has two subtypes: if a single copy of the $\alpha$ allele sufficiently suppresses the toxin expression of two $\beta$ alleles (and vice versa), $\alpha$ and $\beta$ are strongly-suppressed lethal alleles; if two copies of $\alpha$ are required to suppress two copies of $\beta$ (and vice versa), they are weakly-suppressed lethals. There are six genotypes in the homologous system and nine genotypes in the two non-homologous systems, see table 1 and appendix B (where the relative fitnesses for all genotypes are given explicitly for both sexes). The genetics are linked to a continuous time ecological model of the mosquito lifecycle and a coupled Ross-MacDonald epidemiological model of a vector-borne disease (see Figure 1 for a schematic overview of this modelling framework). We consider the use of underdominance only as a method of vector population suppression, and disregard the possibility of linking payload genes to the engineered underdominance constructs. 


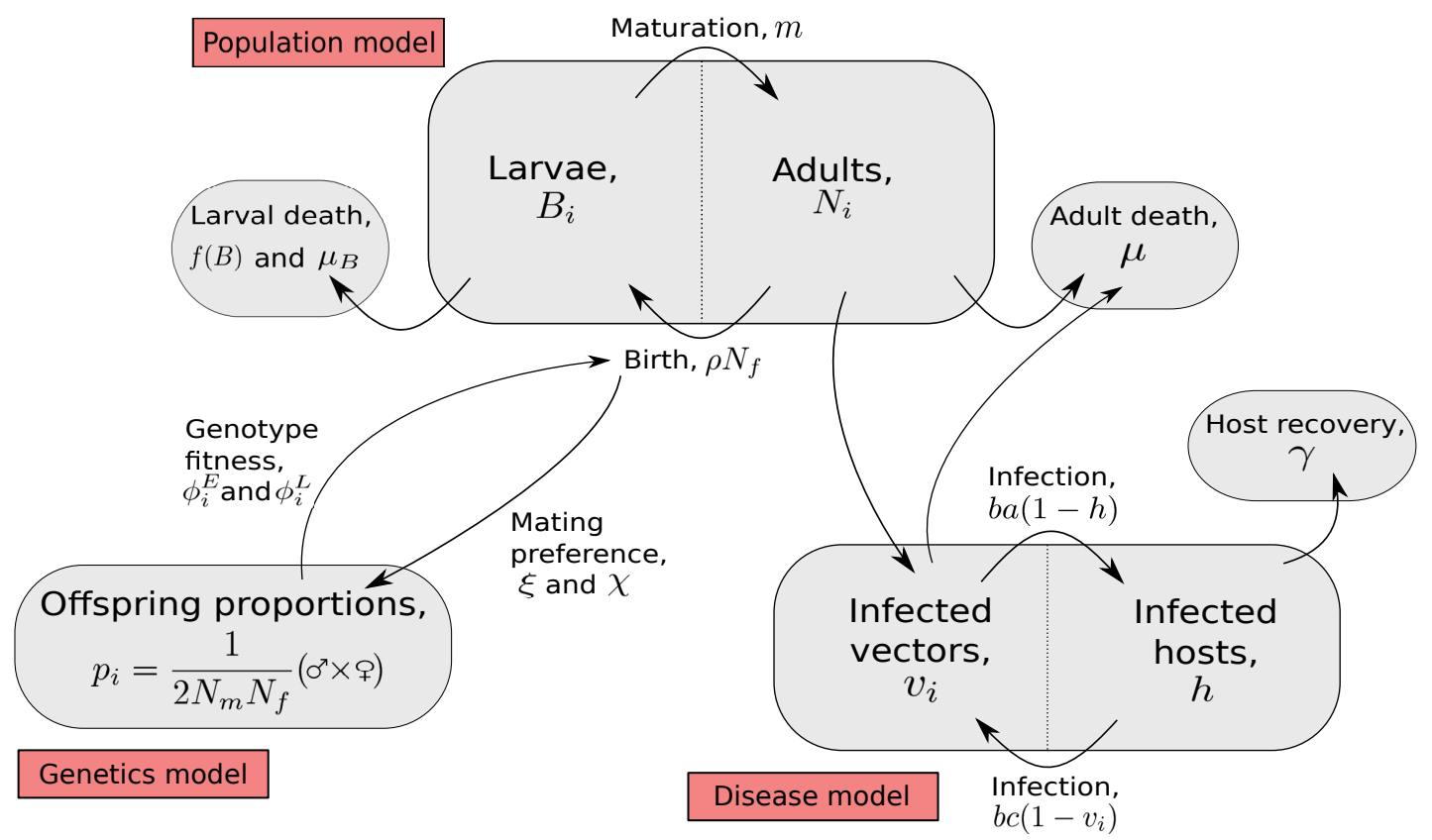

Figure 1: Schematic of the mathematical model defined in (2.1) and (2.9). Arrows depict how variables feed into one another (e.g. larvae feed into adults) or into 'sinks' (e.g. larvae feed into the larval mortality sink). Arrow labels show the parameters that govern the flow between variables. The details of the genetics model, including the implementations of mating preference and genotype fitness, are given in appendix A and appendix B. Parameter and variable definitions are given in table 2 and table 3 , respectively.

\subsection{Population dynamics}

We propose a stage-structured population model for the mosquito, taking into account an aquatic juvenile stage $(B)$ and an adult stage $(N)$. The dynamics are captured by the following set of ordinary differential equations

$$
\begin{aligned}
\frac{\mathrm{d} B_{i}}{\mathrm{~d} t} & =\rho p_{i} N_{f} \phi_{i}^{E}-\left(f_{i}(B)+m+\mu_{B}\right) B_{i}, \\
\frac{\mathrm{d} \hat{B}_{i}}{\mathrm{~d} t} & =\rho p_{i} N_{f} \hat{\phi}_{i}^{E}-\left(f_{i}(B)+m+\mu_{B}\right) \hat{B}_{i}, \\
\frac{\mathrm{d} N_{i}}{\mathrm{~d} t} & =m B_{i} \phi_{i}^{L}-\mu N_{i}, \\
\frac{\mathrm{d} \hat{N}_{i}}{\mathrm{~d} t} & =m \hat{B}_{i} \hat{\phi}_{i}^{L}-\mu \hat{N}_{i}
\end{aligned}
$$

where $\rho$ is the oviposition rate of the adult females, $p_{i}$ is the proportion of individuals of each sex that arise from the $i^{t h}$ genetic mating cross (see appendix A), $m$ is the larval maturation rate and $\mu$ and $\mu_{B}$ are the density-independent mortality rates for adults and juveniles, respectively. The relative fitness of a female (male) of genotype $i, \phi_{i}\left(\hat{\phi}_{i}\right)$, is constructed multiplicatively from the contributions of each of the alleles that make up the genotype (with wild-type alleles contributing a relative fitness of unity). Thus,

$$
\phi_{i}=\kappa_{\mathrm{a}}^{m_{i}} \kappa_{\ell}^{n_{i}},
$$

where $m_{i}$ is the total number of trangenes carried by genotype $i$ (imposing an ambient cost $c_{\mathrm{a}}$ each) and $n_{i}\left(\leq m_{i}\right)$ is the number of unsuppressed toxin transgenes carried (imposing a partially lethal cost $c_{\ell}$ each), with $\kappa_{\mathrm{a}}=1-c_{\mathrm{a}}$ and $\kappa_{\ell}=1-c_{\ell}$. The relative fitnesses $\phi_{i}^{E}$ and $\phi_{i}^{L}$ are mutually exclusive (see below) and account for early- and late-acting fitness costs, respectively. Hats on variables denote male versions that may differ from the female version-for example $\hat{\phi}_{i} \neq \phi_{i}$ 
if the heterozygote lethality is female-specific (e.g. table 1). The function $f_{i}(B)$ in $(2.1 \mathrm{a})$ and $(2.1 \mathrm{~b})$ accounts for density-dependent mortality at the larval stage, and due to the lack of robust evidence on this ecological process we choose to use the flexible function [30]

$$
f(B)=\ln \left[1+(\nu B)^{\eta}\right]
$$

where $\eta$ is the strength of density dependence and $\nu$ is the scale parameter of the density-dependent effects. We define $\nu$ such that the following relation holds:

$$
\nu=\frac{m}{2 k^{*} H \mu}\left(\mathrm{e}^{\left\{\rho m /(2 \mu)-m-\mu_{B}\right\}}-1\right)^{\frac{1}{\eta}},
$$

to ensure that the equilibrium wild vector population $N_{1}^{*}$ is equivalent to $k^{*} H$, the number of wild-type vectors per host at equilibrium $\left(k^{*}\right)$ multiplied by the host population ( $H$, assumed constant). The total population sizes are given by

$$
B=\sum_{j=1}^{n_{g}}\left(B_{j}+\hat{B}_{j}\right), \quad N_{f}=\sum_{j=1}^{n_{g}} N_{j}, \quad N_{m}=\sum_{j=1}^{n_{g}} \hat{N}_{j},
$$

where we sum over all genotypes (including both males and females in the larval population $B$ ), of which there are $n_{g}$. For early-acting lethality, the fitness costs act at reproduction (i.e. in (2.1a) and (2.1b)), thus $\phi_{i}^{E}=\phi_{i}, \phi_{i}^{L}=1, \hat{\phi}_{i}^{E}=\hat{\phi}_{i}$ and $\hat{\phi}_{i}^{L}=1$; for late-acting lethality, the fitness costs act at maturation to the adult stages (i.e. in (2.1c) and (2.1d)), thus $\phi_{i}^{E}=1, \phi_{i}^{L}=\phi_{i}, \hat{\phi}_{i}^{E}=1$ and $\hat{\phi}_{i}^{L}=\hat{\phi}_{i}$.

\begin{tabular}{lcccc|cccc}
\hline & \multicolumn{4}{c}{ Genotype } & \multicolumn{2}{c}{ Female } \\
Lethality & $A A$ & $A \alpha, A \beta$ & $\alpha \beta$ & $\alpha \alpha, \beta \beta$ & $A A$ & $A \alpha, A \beta$ & $\alpha \beta$ & $\alpha \alpha, \beta \beta$ \\
\hline BSL & 1 & $\kappa_{\mathrm{a}} \kappa_{\ell}$ & $\kappa_{\mathrm{a}}^{2}$ & $\kappa_{\mathrm{a}}^{2} \kappa_{\ell}^{2}$ & 1 & $\kappa_{\mathrm{a}} \kappa_{\ell}$ & $\kappa_{\mathrm{a}}^{2}$ & $\kappa_{\mathrm{a}}^{2} \kappa_{\ell}^{2}$ \\
FSL & 1 & $\kappa_{\mathrm{a}}$ & $\kappa_{\mathrm{a}}^{2}$ & $\kappa_{\mathrm{a}}^{2}$ & & & \\
\hline
\end{tabular}

Table 1: Genotype fitness for the homologous engineered underdominance system for bi-sex lethality (BSL) and femalespecific lethality (FSL); $\kappa_{\mathrm{a}}=1-c_{\mathrm{a}}$ and $\kappa_{\ell}=1-c_{\ell}$ where $c_{\mathrm{a}}$ is the ambient fitness cost of carrying a single transgene and $c_{\ell}$ is the (partially) lethal fitness cost of carrying an unsuppressed toxin gene. Fitness costs are assumed to combine multiplicatively. In particular, the engineered homozygote fitness is $\phi_{\mathrm{hom}}=\left(1-c_{\mathrm{a}}\right)^{2}$ and the heterozygote fitness is $\phi_{\text {het }}=\left(1-c_{\mathrm{a}}\right)\left(1-c_{\ell}\right)$. Genotypes with the same fitness are grouped. For the non-homologous underdominant systems see tables B.4-B.7 in appendix B.

\subsection{Disease dynamics}

The basic disease dynamics follow a modified Ross-MacDonald approach [31] such that if the density of infected mosquitoes is

$$
\frac{d Y}{d t}=b c\left(N_{f}-Y\right) h-\mu Y
$$

where each susceptible mosquito (of which there are $N_{f}-Y$ ) bites $b$ people per day of which a proportion $h$ are infectious and fraction $c$ of those bites acquires infection then the proportion of infected vectors $v$ is:

$$
\frac{\mathrm{d} v}{\mathrm{~d} t}=b c(1-v) h-\left(\mu+\frac{1}{N_{f}} \frac{\mathrm{d} N_{f}}{\mathrm{~d} t}\right) v
$$


The proportion of infected humans is:

$$
\frac{\mathrm{d} h}{\mathrm{~d} t}=\frac{N}{H} b a(1-h) v-\gamma h,
$$

where $a$ is the proportion of bites on susceptible humans by infectious mosquitoes that result in infection and $\gamma$ is the host recovery rate. The dynamics of the expanded vector-disease interaction when GM mosquitoes are released are:

130

$$
\begin{aligned}
\frac{\mathrm{d} h}{\mathrm{~d} t} & =\frac{1}{H} b a(1-h) \sum_{i=1}^{n_{g}} N_{i} v_{i}-\gamma h, \\
\frac{\mathrm{d} v_{i}}{\mathrm{~d} t} & =b c\left(1-v_{i}\right) h-\left(\mu+\frac{1}{N_{i}} \frac{\mathrm{d} N_{i}}{\mathrm{~d} t}\right) v_{i},
\end{aligned}
$$

where the adult females in every genotype can be a vector of disease. Parameter definitions and values for 134 the epidemiological model (2.9) and the population model (2.1) are listed in table 2; variable definitions 135 are given in table 3. 


\begin{tabular}{|c|c|c|c|}
\hline Parameter & Description & Default value & Notes \\
\hline$\rho$ & per adult female oviposition rate & 16 per day & [32-34] \\
\hline$m$ & larval maturation rate & 0.1 & {$[35]$} \\
\hline$\mu$ & adult mosquito death rate & $\ln \frac{10}{9}$ per day & [36-40], chosen to be conservative \\
\hline$\mu_{B}$ & $\begin{array}{l}\text { density-independent larval death } \\
\text { rate }\end{array}$ & 0.03 per day & {$[41]$} \\
\hline$\eta$ & strength of density dependence & 0.9 & $\begin{array}{l}\text { varies from } \eta<1 \text { for contest to } \eta> \\
1 \text { for scramble }\end{array}$ \\
\hline$\nu$ & scale of larval density-dependence & eq. (2.4) & ensures $N_{1}^{*}=k^{*} H$ \\
\hline$c_{\mathrm{a}}$ & $\begin{array}{l}\text { ambient fitness cost of carrying } \\
\text { single transgene }\end{array}$ & variable & $\begin{array}{l}\text { fitness cost acts even when the } \\
\text { toxin is suppressed }\end{array}$ \\
\hline$c_{\ell}$ & $\begin{array}{l}\text { lethal fitness cost of carrying un- } \\
\text { suppressed toxin gene }\end{array}$ & variable & lethality may not be totally efficient \\
\hline$\kappa_{\mathrm{a}}$ & $\begin{array}{l}\text { relative fitness derived from in- } \\
\text { curring cost } c_{\mathrm{a}}\end{array}$ & $1-c_{\mathrm{a}}$ & relative to wild type \\
\hline$\kappa_{\ell}$ & $\begin{array}{l}\text { relative fitness derived from in- } \\
\text { curring cost } c_{\ell}\end{array}$ & $1-c_{\ell}$ & relative to wild type \\
\hline$\phi_{i} / \hat{\phi}_{i}$ & $\begin{array}{l}\text { female/male relative fitness of } \\
\text { genotype } i\end{array}$ & $\kappa_{\mathrm{a}}^{n} \kappa_{\ell}^{m}$ & $\begin{array}{l}n, m \text { are genotype-specific numbers } \\
\text { of transgenes imposing costs } c_{\mathrm{a}}, c_{\ell}\end{array}$ \\
\hline$H$ & host population & 1000 & variable \\
\hline$k^{*}$ & vectors per host at equilibrium & 2 & $\begin{array}{l}\text { but can be as high as } 200[39,42- \\
44]\end{array}$ \\
\hline$b$ & mosquito bite rate & 2.8 per day & {$[45]$} \\
\hline$a$ & $\begin{array}{l}\text { vector to human transmission ef- } \\
\text { ficiency }\end{array}$ & 0.084 & median value from $[36,37]$ \\
\hline$c$ & $\begin{array}{l}\text { human to vector transmission ef- } \\
\text { ficiency }\end{array}$ & 0.216 & median value from $[36,37]$ \\
\hline$\gamma$ & host recovery rate & $1 / 14$ per day & $\begin{array}{l}\text { variable, assuming a two-week av- } \\
\text { erage }\end{array}$ \\
\hline$\xi$ & $\begin{array}{l}\text { mating preference of wild females } \\
\text { for wild males }\end{array}$ & variable & $\begin{array}{l}\text { the proportion of wild females from } \\
\text { each encounter with non-wild males } \\
\text { that instead choose to mate with } \\
\text { wild males }\end{array}$ \\
\hline$\chi$ & $\begin{array}{l}\text { increase in wild-wild matings due } \\
\text { to mating preference }\end{array}$ & $\frac{\xi}{\hat{N}_{1}} \sum_{i=2}^{n_{g}} \hat{N}_{i}$ & $\begin{array}{l}\text { where } \hat{N}_{i} \text { is the male population of } \\
\text { genotype } i, i \in\left[1, n_{g}\right] \text { with } i=1 \\
\text { being wild-type }\end{array}$ \\
\hline
\end{tabular}

Table 2: Parameter definitions and values. Anopheles gambiae is used as the model species, with the disease model parameterised using malaria data.

\begin{tabular}{cl}
\hline Variable & Description \\
\hline$B_{i}, \hat{B}_{i}$ & female and male larvae population of genotype $i$ \\
$N_{i}, \hat{N}_{i}$ & female and male adult mosqito population of genotype $i$ \\
$h$ & porportion of human hosts infected \\
$v_{i}$ & proportion of adult female mosquitoes of genotype $i$ infected \\
\hline
\end{tabular}

Table 3: Definitions of the variables for which we solve as functions of time, $t$, using the coupled dynamical system defined in $(2.1)$ and (2.9).

\section{Results: genetics}

In this section we examine different genetic configurations for UD gene drives: bi-sex and maleonly releases; variation in fitness costs imposed by the engineered alleles; and female-specific and bi-sex lethality. We use two metrics to aid in the analysis: (i) the threshold frequency that a single initial release must reach in order to trigger the UD drive in favour of the engineered constructs; (ii) the effect on the disease burden of both successful and unsuccessful attempts to trigger the UD drive, quoted as the 
percentage of cases averted over a time period compared to a control-free scenario. The number of cases averted is approximated by ([mean no. with no releases - mean no. with releases]/average duration of disease $) \times$ no. of days). The percentage of cases averted is then calculated with respect to the control-free scenario.

We define a function, $\Gamma(t)$ as an indicator of whether the UD drive has been successfully triggered after a time $t$ has passed:

$$
\Gamma(t)=\frac{N_{1}(t)-N_{\mathrm{E}}(t)}{\sum_{i=1}^{n_{g}} N_{i}(t)}
$$

where $N_{1}$ is the number of female wild-type homozygotes and $N_{\mathrm{E}}$ is the number of female engineered homozygotes. Prior to any releases, $\Gamma=1$, then, as engineered homozygotes are released, $\Gamma$ will decrease. Eventually, $\Gamma$ will stabilise either at $\Gamma=1$ (transgenic insects die out) if the UD threshold was not reached, or $\Gamma \rightarrow-1$ (transgenic insects spread to fixation) if the threshold was successfully reached (the limits may not be reached exactly due to heterozygotes surviving in small numbers). The amount of time that is sufficient to be sure that an attempt to trigger the UD drive has been either a success or a failure is dependent upon how close to the threshold the initial release was: a release very close to the threshold will take a longer time to either fade out or to spread to fixation. Figures $2 \mathrm{a}-2 \mathrm{c}$ show that after 360 days $\Gamma$ has converged to an approximation of a step function (where the release frequency $f$ has been discretised at the third decimal place), crossing quickly from $\Gamma \approx 1$ to $\Gamma \approx-1$. We choose to use the time horizon of one year $\left(t_{N}=365\right.$ days) in all computations henceforth to ensure that the threshold release frequency has been accurately determined (longer simulations will not provide richer information about the threshold). We use the term release frequency to mean the proportion of the entire population (of the same sex as the released insects) that are engineered homozygotes immediately after an initial release of a given size. The term threshold release frequency means the lowest release frequency that produces a negative $\Gamma$ value after one year $\left(\Gamma\left(t_{N}\right)<0\right)$. The conversion between release ratio (GM mosquitoes per one wild mosquito of the same sex) and release frequency $f$ is shown in fig. $2 \mathrm{~d}$ : for example, a release frequency of $f=0.5$ translates to releasing one mosquito for every one wild mosquito of the same sex; $f=0.9$ translates to releasing nine mosquitoes for every one wild mosquito of the same sex; $f=0.99$ translates to releasing 99 mosquitoes for every one wild mosquito of the same sex.

\subsection{Male-only releases}

Mosquito vector control interventions generally release males to disrupt a wild population. Previous theoretical studies of underdominance as a population control technique have generally not considered the problem of sex, considering instead genderless genetic models or releases composed of both sexes. This neglects the stumbling block of releasing biting insects and potential vectors of disease.

We find that male-only releases require a higher threshold to be reached than bi-sex releases, for both the homologous and non-homologous UD systems (compare figs. 3a and 3b). This is due to the requirement for male releases to establish a self-sustaining population: that heterozygotes survive to breed in order to produce female engineered homozygotes. The strongly-suppressed non-homologous UD 


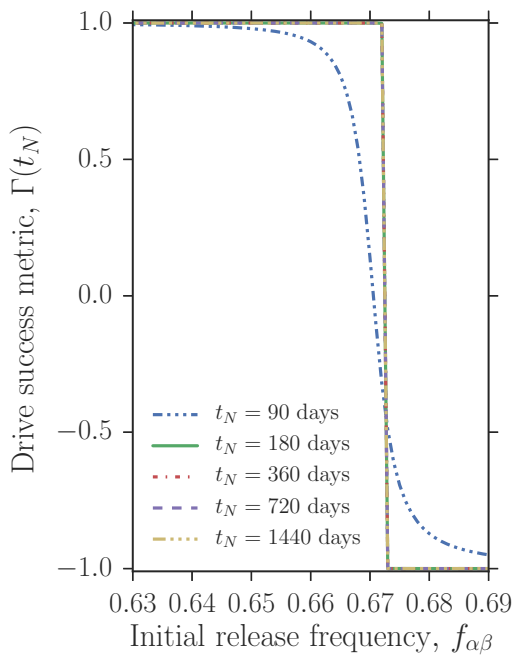

(a)

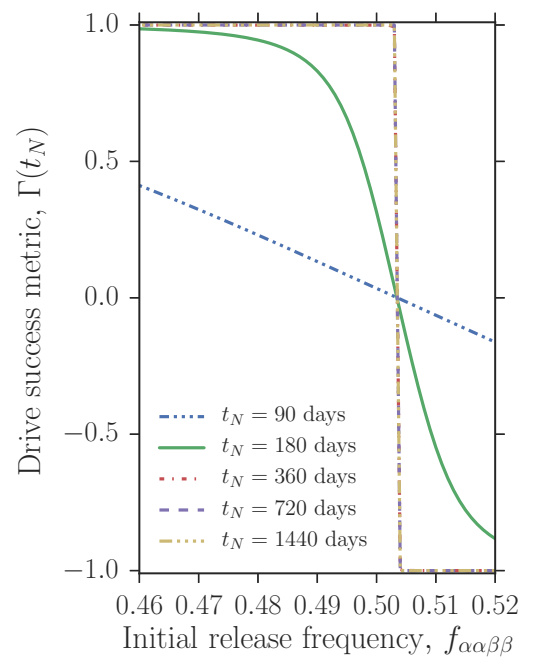

(b)

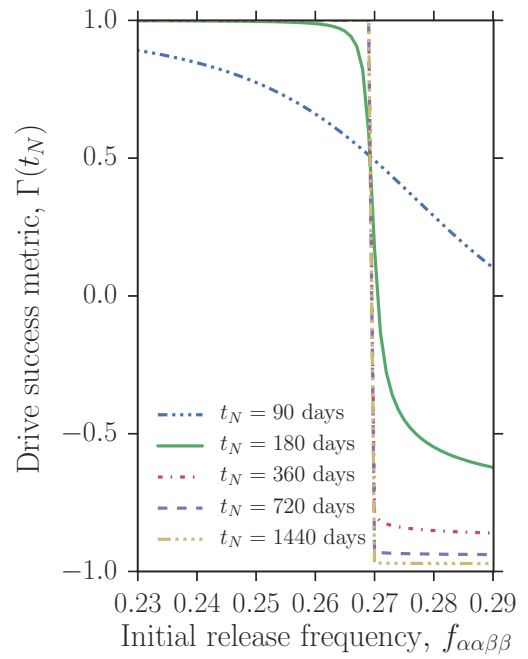

(c)

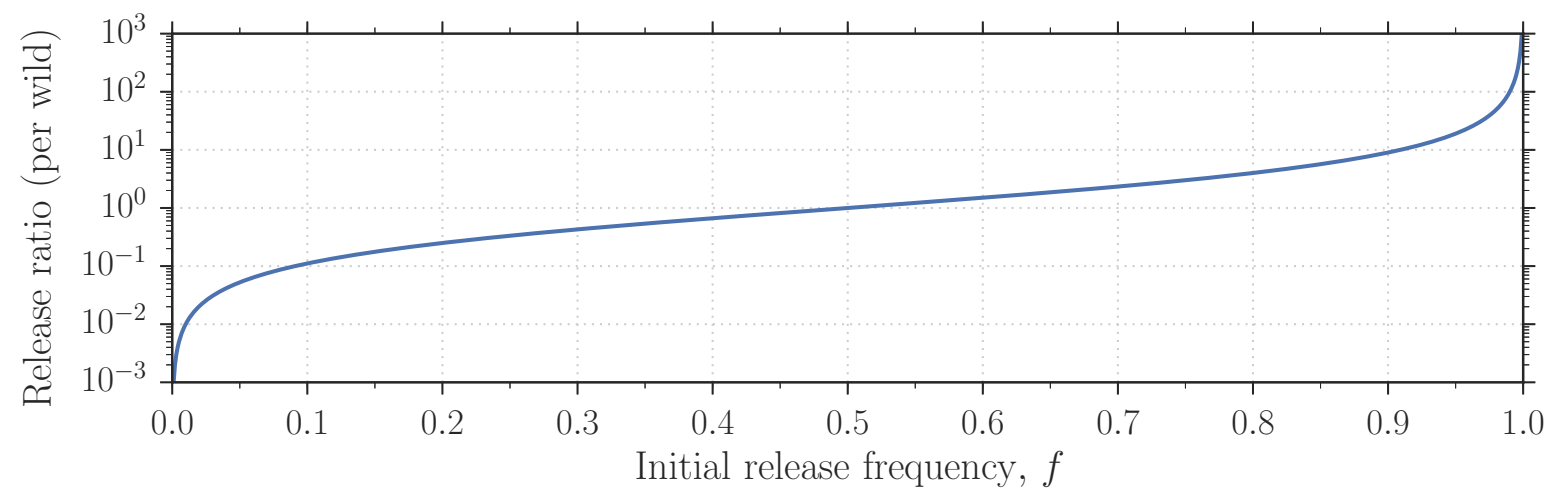

(d)

Figure 2: The threshold frequency is accurately resolved by running simulations for a time horizon of $t_{N} \geq 360$ days, as $\Gamma\left(t_{N}\right)$ tends towards a step function changing between $\Gamma\left(t_{N}\right) \approx 1$ (wild-type dominant) and $\Gamma\left(t_{N}\right) \approx-1$ (engineered genotypes dominant), centred on the threshold release frequency. Plots show (a) homologous, (b) weakly-suppressed non-homologous and (c) strongly-suppressed non-homologous underdominance, for a bi-sex release with early-acting bi-sex lethality. (d) The conversion between initial release frequency and number of transgenic mosquitoes released for every one wild mosquito of the same sex, calculated using $n_{p w}=f /(1-f)$, where $n_{p w}$ is the number released per wild-type of the same sex. Parameters as in table 2 with fitness $\operatorname{costs} c_{\mathrm{a}}=0$ and $c c_{\ell}=0.99$. 


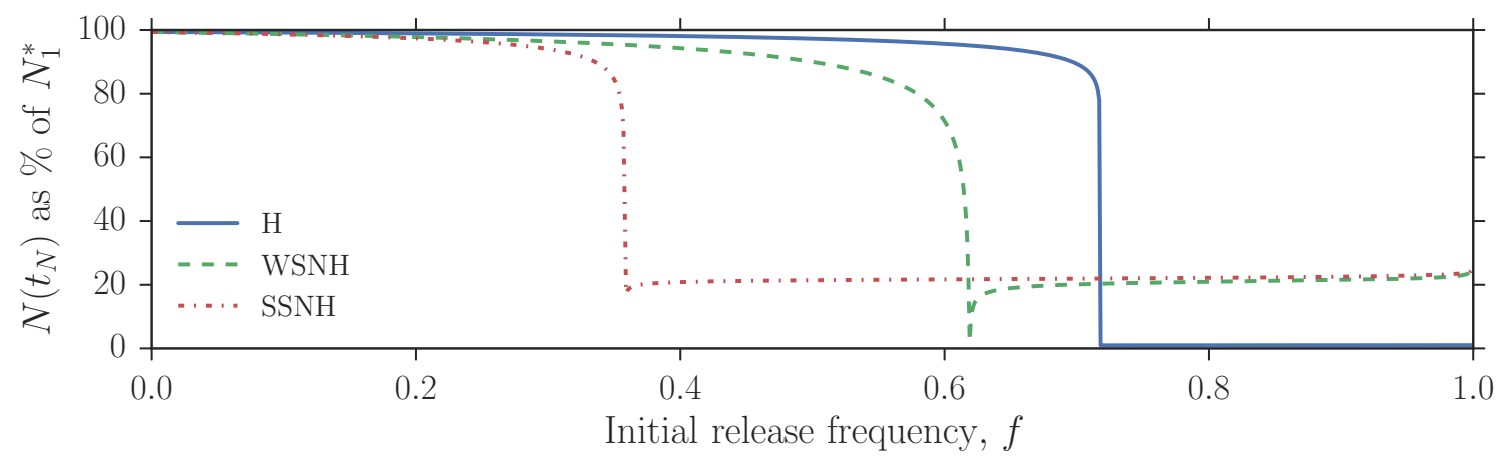

(a) Bi-sex release

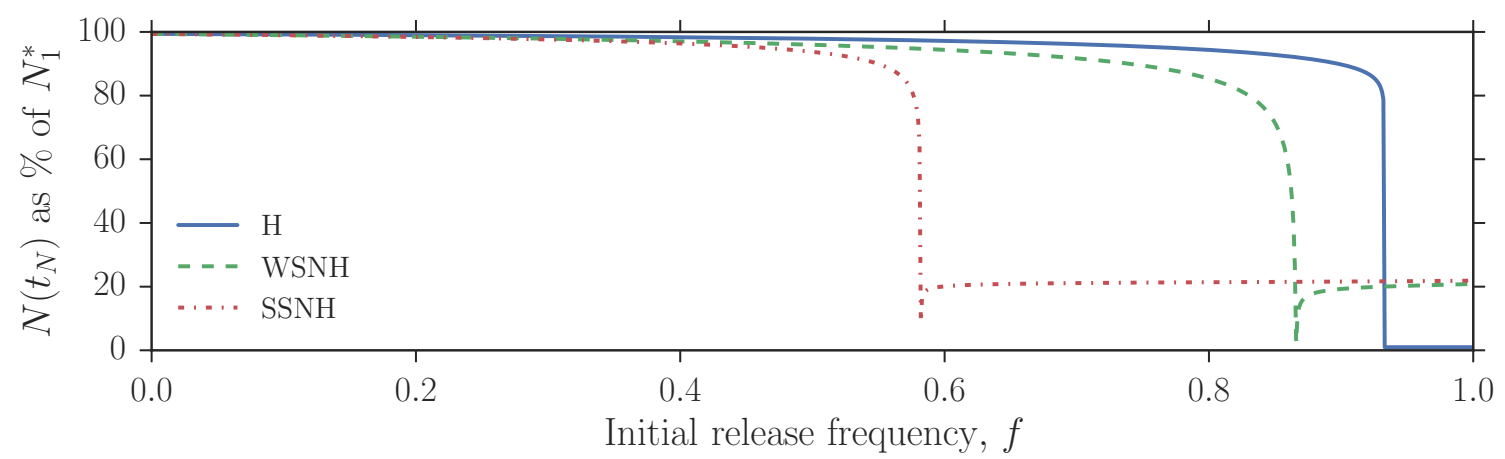

(b) Male-only release

Figure 3: Using male-only rather than bi-sex releases increases the required release threshold for a successful underdominance drive, but only marginally alters the outcome of a successful drive. Plots show the total vector population $N\left(t_{N}\right)$ at the end of one year $\left(t_{N}=365\right.$ days $)$ as a percentage of the wild-type female equilibrium population, $N_{1}^{*}$, as the initial release frequency is varied, for the homologous $(\mathrm{H})$, weakly-suppressed non-homologous (WSNH), and strongly-suppressed nonhomologous (SSNH) underdominance systems. Top plot (a) shows a bi-sex release; bottom plot (b) shows a male-only release. Genetic fitness costs are $c_{\mathrm{a}}=0.05, c_{\ell}=0.9$. Other parameters as in table 2, for an initial release with early-acting bi-sex lethality.

system has the lowest threshold frequency; the homologous system has the highest threshold release ratio.

The population suppression caused by the non-homologous UD systems peaks (strongly) when releasing exactly at the threshold frequency (fig. 3). The release of 'extra' mosquitoes partly counteracts the population suppression that occurs in the transition period between wild-type fixation and transgenic fixation.

The homologous UD system generally leads to greater population suppression than the two nonhomologous UD systems (due to having fewer viable genotypes), almost eradicating the active vector population (fig. 3); this system acts more in the spirit of 'removal' than replacement, leaving the 'replaced' population greatly diminished. Conversely, the non-homologous systems may be useful for population replacement (though they also enact substantial suppression when $c_{\mathrm{a}}>0$, e.g. fig. $3 \mathrm{~b}$, and the level of suppression is strongly dependent on $c_{\mathrm{a}}$ ).

\subsection{Effects of genetic fitness costs}

An underdominance system has two forms of genetic fitness cost: (i) the desirable toxic fitness cost responsible for lethality in heterozygotes and (ii) the ambient cost on individuals carrying a transgene that could arise, for example, through incomplete suppression of toxin expression due to imperfect binding of the suppressor protein to the promoter [25] ('leaky' toxicity), or through other unforeseen genetic complications. 
It is possible to achieve an underdominance drive with a relatively small toxic transgene fitness cost $\left(c_{\ell}\right)$ and changes in the heterozygote fitness affect the UD threshold only slightly (fig. 4a). However, a high heterozygote relative fitness (i.e. low $c_{\ell}$ ) adversely affects the resulting disease suppression of a successfully triggered UD drive (fig. 4c). Perfect or near-perfect lethality is detrimental to the UD drive process for a male-only release, as in order to establish a population of female transgenic homozygotes ( $\alpha \beta$ or $\alpha \alpha \beta \beta$ for homologous or non-homologous UD, respectively), the heterozygote offspring of engineered males and wild-type females must survive and mate. It is worth noting that the perfectly lethal underdominant system initially proposed by Davis et al. [25] would be impossible to engineer: in breeding the transgenic line, the first transgenic insect would necessarily be heterozygote and hence would be nonviable.

Fitness costs on the engineered homozygotes (through 'leaky' toxicity causing $c_{\mathrm{a}}>0$, for example) can increase the drive threshold significantly (fig. 4b). The non-homologous UD systems are particularly affected, with the threshold frequency overtaking that of the homologous system at $c_{\mathrm{a}} \approx 0.1$ for the weakly-suppressed system and $c_{\mathrm{a}} \approx 0.24$ for the strongly-suppressed system (fig. $4 \mathrm{~b}$ ). Interestingly, even high $\left(c_{\mathrm{a}}<0.26\right)$ unintended fitness costs associated with genetic modification are associated with a higher percentage of cases averted (fig. 4d) when a UD drive is successfully triggered at its threshold (above $c_{\mathrm{a}} \approx 0.26$ the threshold frequencies exceed $f=0.999$, a release ratio of around $1000: 1$, which we define here as 'out of bounds' due to being practically unattainable). Increasing $c_{\mathrm{a}}$ has only a marginal effect on the percentage of cases averted by the homologous system (fig. 4d).

We also examined the effect of early vs late-acting lethality (i.e. whether the fitness costs are incurred in the egg or pupal stage) on the efficacy of the control. We found that the UD threshold is insensitive to the timing of the heterozygote lethality, but late-acting lethality has a marginally greater impact on the resulting disease burden.

\subsection{Female-specific lethality}

Many genetic constructs in development have sex-specific action, such as X-shredders [46, 47] (which act to distort the offspring sex ratio), female killing or sterilising alleles [48-50] and female-specific flightless phenotype transgenes [51,52] (which cause death indirectly via flightlessness). Female-specific lethal (FSL) systems are in principle more efficient than standard SIT [48], and may have benefits related to resistance management when used in an integrated vector management programme [53, 54].

Using a construct that kills only females has interesting effects in an underdominance system. First, the UD drive threshold is increased when compared to a bi-sex lethal (BSL) gene (fig. 5a), in particular for the homologous UD system for which the threshold release ratio is more than doubled from $33: 2$ for BSL to $89: 2$ for FSL when $c_{\mathrm{a}}=0.1$. Second, the disease suppression that is achieved by an FSL release is generally greater than for a BSL release of the same initial release frequency (fig. 5b), except in narrow regions where the BSL threshold has been met, triggering a successful UD drive, but the release frequency is still below the FSL threshold. Importantly, at release frequencies below the threshold for both FSL and BSL systems, FSL releases avert a higher percentage of disease cases over the year than do BSL releases (fig. 5b). 


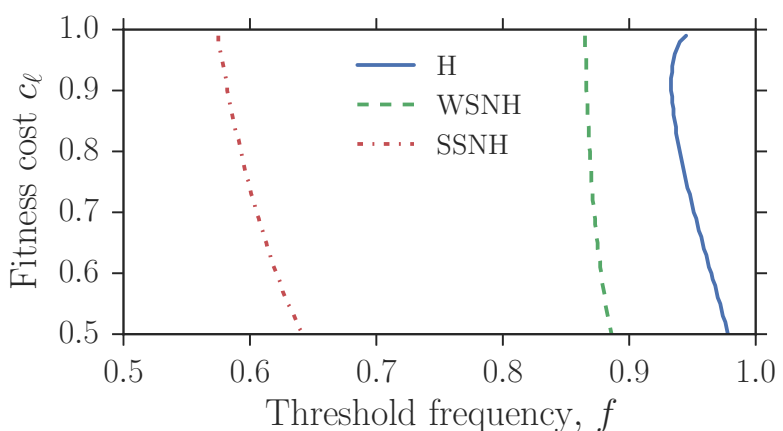

(a)

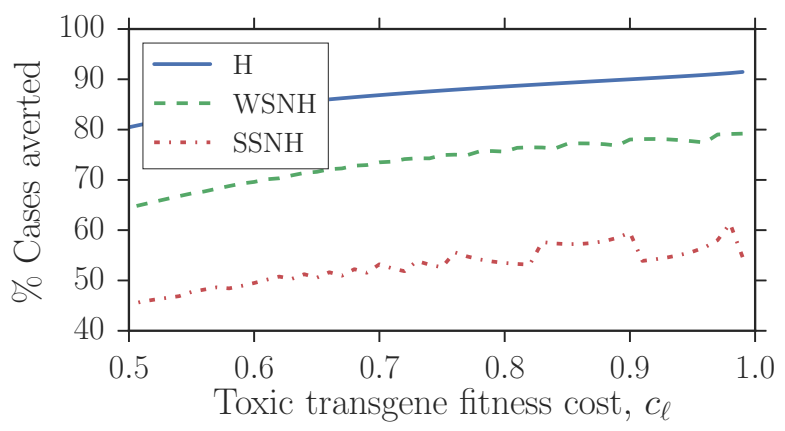

(c)

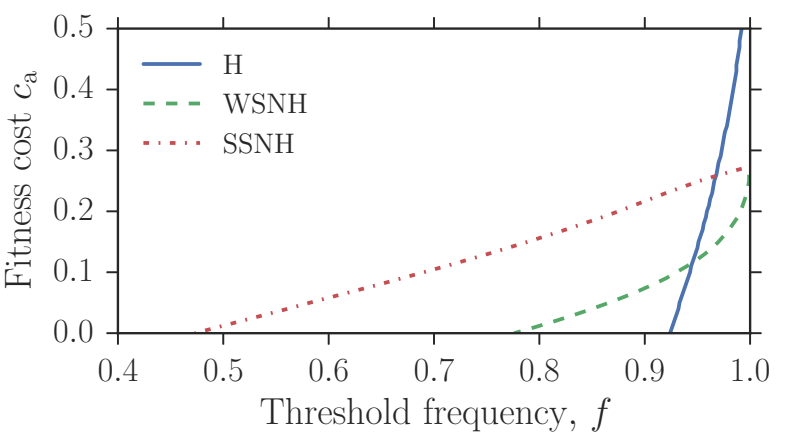

(b)

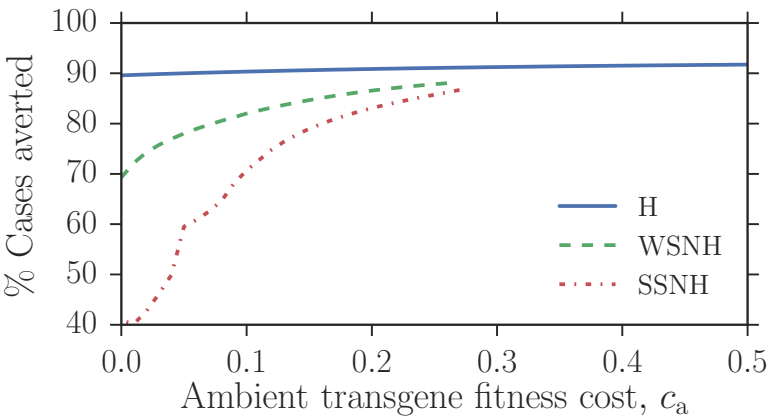

(d)

Figure 4: The performance of a control effort using non-homologous engineered underdominance is influenced greatly by the ambient transgene fitness cost, $c_{\mathrm{a}}$, and to a lesser extent by the toxic transgene fitness cost, $c_{\ell}$. The homologous underdominance system is less sensitive to changes in the transgene fitness costs. Top row shows the threshold release frequency when changing the (a) toxic and (b) ambient transgene fitness costs for the homologous (H), weakly-suppressed non-homologous (WSNH), and strongly-suppressed non-homologous (SSNH) underdominance systems. Bottom row shows the percentage of cases averted over one year for a given (c) toxic and (d) ambient transgene fitness cost when a single release is made at the threshold frequency. In (c) $c_{\mathrm{a}}=0.05$; in (d) $c_{\ell}=0.9$. Other parameters as in table 2 , for initial releases with early-acting bi-sex lethality.

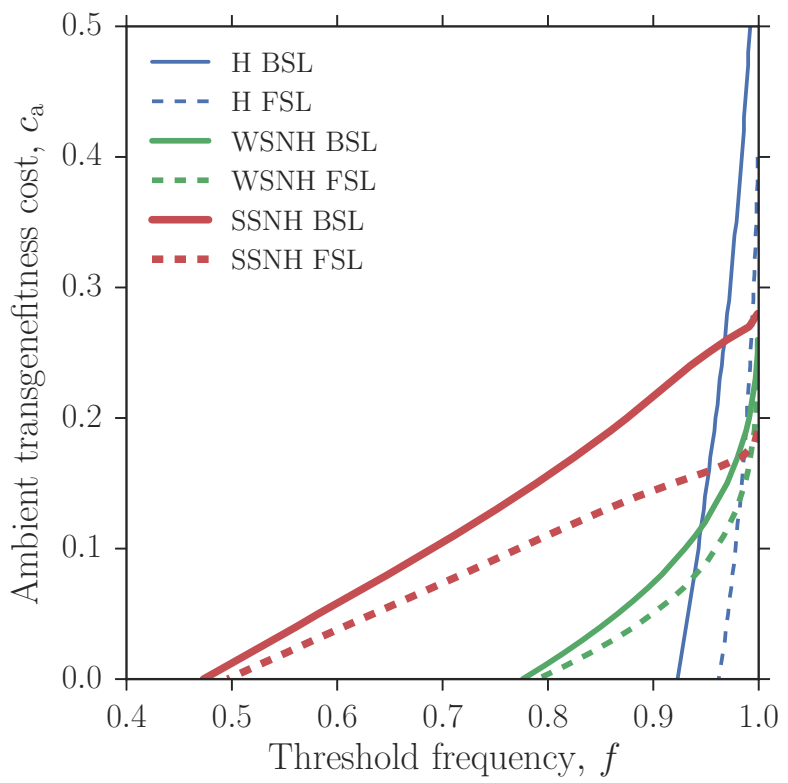

(a)

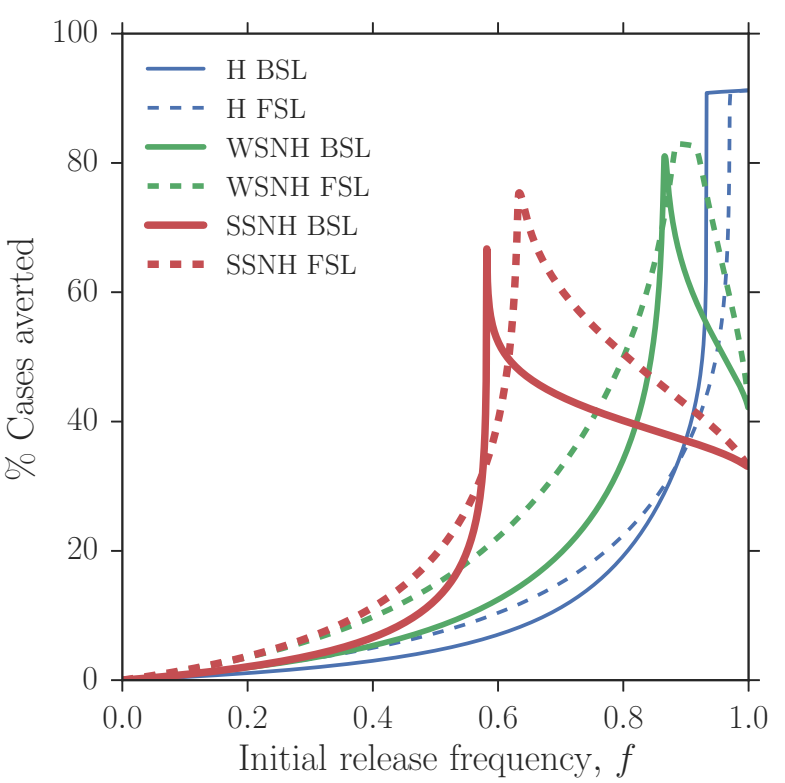

(b)

Figure 5: Female-specific lethality increases the underdominance threshold over that of bi-sex lethality, but has a greater impact on the disease burden for both successful and unsuccessful attempts to trigger the underdominance drive. (a) The underdominance threshold frequency as $c_{\mathrm{a}}$ is varied with $c_{\ell}=0.9$ held constant, for homologous $(\mathrm{H})$ and weakly-suppressed non-homologous (WSNH) and strongly-suppressed non-homologous (SSNH) underdominance systems that employ femalespecific lethality (FSL), or bi-sex lethality (BSL). (b) Percentage of cases averted during the year compared to the control-free scenario, as initial release frequency $f$ is varied, with $c_{\mathrm{a}}=0.05$ and $c_{\ell}=0.9$. Parameters as in table 2 , for a male-only initial release with late-acting lethality. 


\section{Results: ecology}

\subsection{Mating preference}

We model the mating success of transgenic mosquitoes as the level of phenotypic preference of wild females to mate proportionally more with wild males than with any other genotype (see appendix A.2). Figure 6 a shows that modest female mating preference can have a large effect on the UD threshold: if one in five wild females chooses to mate with a wild male rather than any other heterozygote or homozygote genotype (an $80 \%$ mating success rate for the transgenic genotypes), the threshold release frequency for the homologous system increases to $f_{\alpha \beta}=0.996$, corresponding to the requirement of releasing almost 250 males for every one wild male - a seventeen-fold increase over the random mating case. The weaklysuppressed non-homologous system suffers a two-fold increase in threshold release ratio when mating success is reduced from $100 \%$ to $80 \%$. The strongly-suppressed non-homologous system is most able to tolerate lower mating success (having the most viable genotypes of the systems tested), with the threshold release ratio increasing to only $3: 1$ at $80 \%$ mating success. Stronger mating preference on the part of the wild females for wild males (translating to a smaller percentage mating success for the transgenic males) has a negative impact on the percentage of disease cases averted throughout the year for a single initial release that successfully triggers an underdominance gene drive at its threshold frequency (fig. $6 \mathrm{~b}$ ).

\subsection{Ecological fitness costs}

We wish to distinguish between the genetic fitness cost imposed by the toxins of the $\alpha$ and $\beta$ alleles and changes in life history parameters that could be caused by lab rearing, loss of 'wild' phenotypic behaviours and any manifestation of genetic load caused by the UD constructs that are not directly associated with heterozygote lethality or ambient genetic transgene fitness cost [55-57]. To this end, we consider the effects of an increased scale of larval competition $(\nu)$, slower maturation $(m)$, a higher rate of adult $(\mu)$ and larval $\left(\mu_{B}\right)$ density-independent mortality and a greater strength of density dependence $(\eta)$. These costs are applied to all non-wild genotypes. Importantly, changing ecological parameters for all genotypes equally does not affect the UD threshold, which relies on the relative, rather than absolute, strengths of each genotype (see appendix $\mathrm{C}$ for an analytical investigation). (The implications for disease and population suppression do depend on the absolute values of the life history parameters.)

Increases in the adult mortality rate have the greatest effect on the UD drive threshold (see fig. 7, which shows results for the strongly-suppressed non-homologous system only; the other systems show qualitatively similar behaviour), with a $20 \%$ increase in $\mu$ above the wild-type value being sufficient to quadruple the required release ratio to over $109: 2$ for the homologous system. The threshold release ratio for the weakly-suppressed non-homologous system more than triples to 20:1; for the strongly-suppressed system the threshold more than doubles to 7:2. The maturation rate and strength of the larval density dependence have a moderate effect on the thresholds. Increasing the density-independent mortality or the scale of the density-dependent mortality of the larvae has only a minor effect on the threshold frequency (fig. 7). 


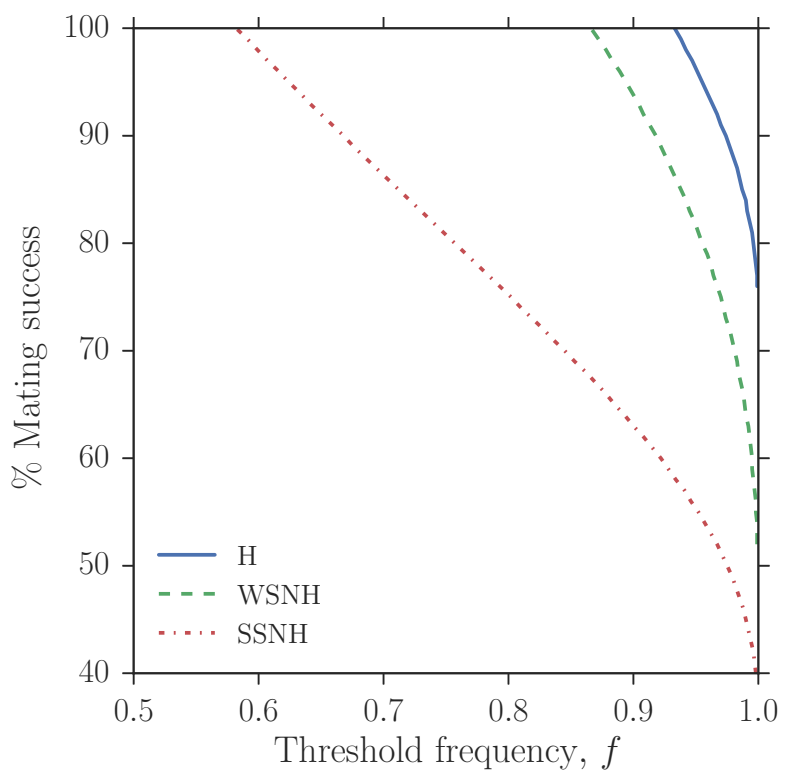

(a)

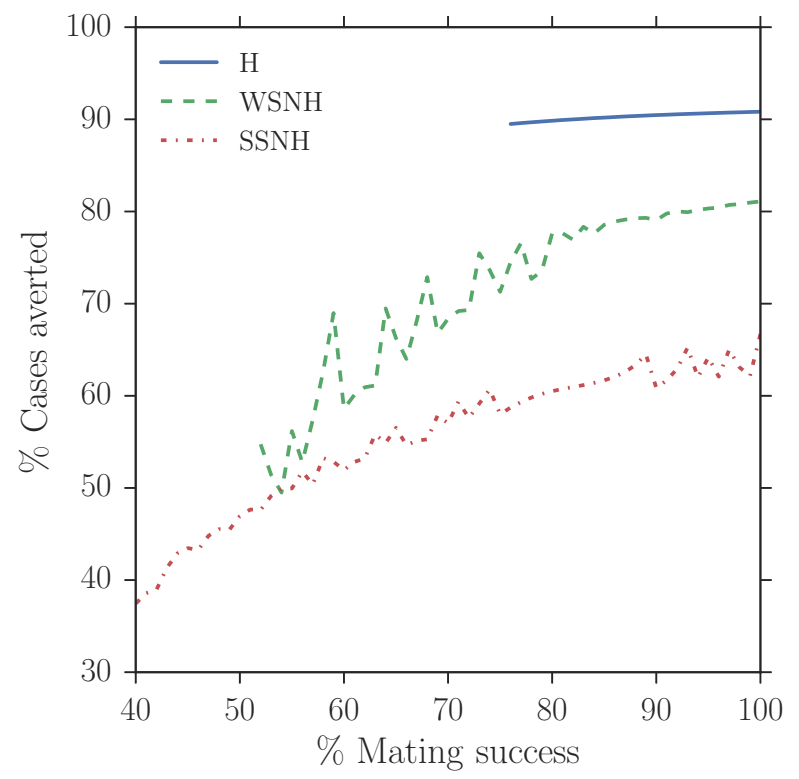

(b)

Figure 6: Reducing the mating success of engineered male mosquitoes with wild females, who proportionally prefer wild males, greatly affects the threshold required to trigger a successful underdominance gene drive. (a) The change in threshold frequency for homologous (H), weakly-suppressed non-homologous (WSNH) and strongly-suppressed non-homologous (SSNH) underdominance systems as the mating success of engineered males with wild females is varied. (b) The effect of mating success on the percentage of cases averted over a year compared with a control-free scenario, when an underdominance drive is successfully triggered by a single release at its particular threshold frequency. Parameters as in table 2 with $c_{\mathrm{a}}=0.05$ and $c_{\ell}=0.9$, for a male-only initial release with late-acting bi-sex lethality. Note, jaggedness in the WSNH and SSNH lines in (b) is due to the sensitivity of these releases to the accuracy with which the threshold frequency is met - the 'narrow region' of efficacy discussed in section 3.1 (see, e.g., sharp troughs in fig. 3). Threshold frequencies are found correct to the third decimal place.

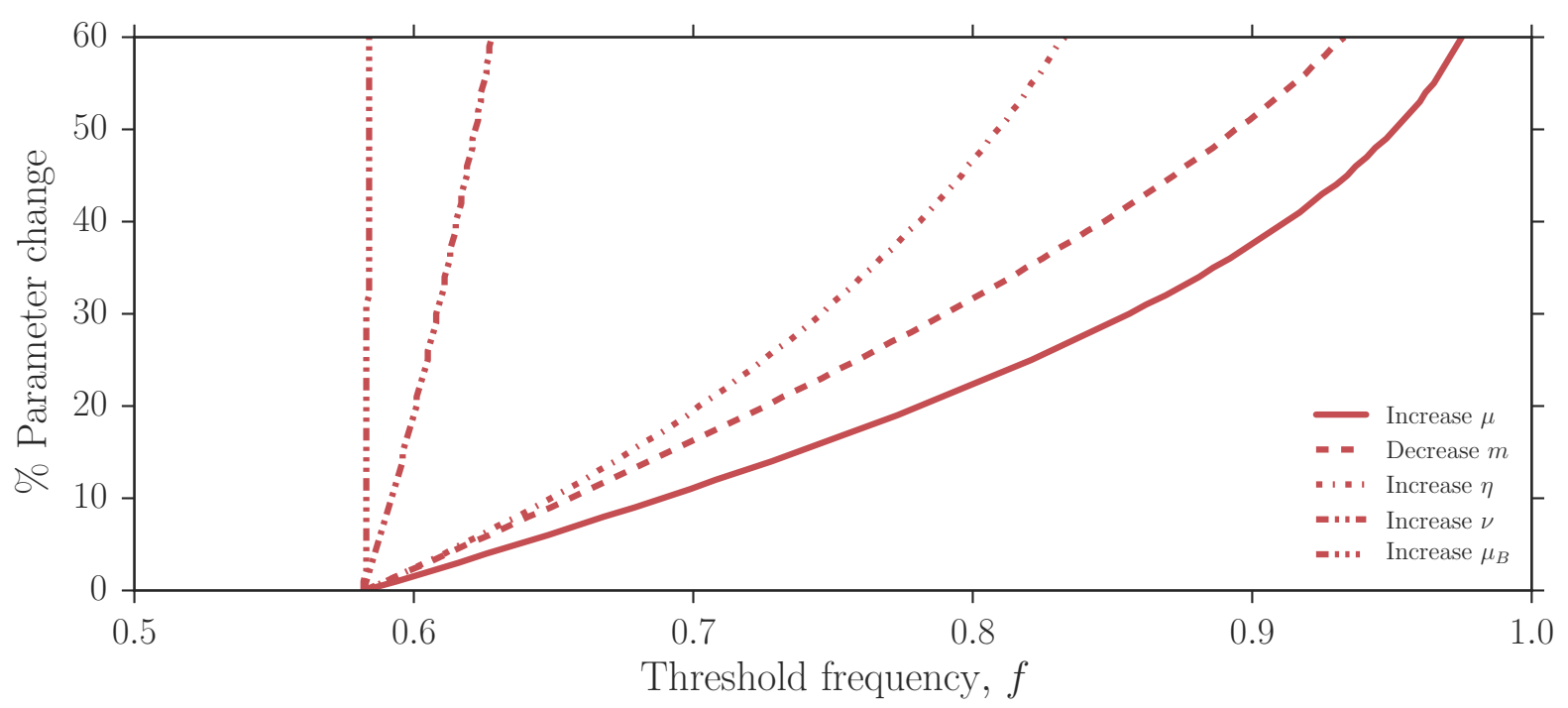

Figure 7: Engineered mosquitoes that have a greater adult mortality rate $(\mu)$ than wild-types face a substantially increased threshold frequency. The larval maturation rate $(m)$ and character (or strength) of the larval density dependence ( $\eta$ ) also play an important role in determining the threshold frequency, while the density-independent larval mortality rate $\left(\mu_{B}\right)$ and scale of density dependence $(\nu)$ play a negligible role. The threshold frequency is plotted as life history parameters of transgene-carrying insects are perturbed, for the strongly-suppressed non-homologous underdominance system. All mosquitoes that contain one or more of the engineered alleles $\alpha$ or $\beta$ are taken to be equally affected by the parameter changes (which change relative to the wild-type value). Unchanged parameters as in table 2 with $c_{\mathrm{a}}=0.05$ and $c_{\ell}=0.9$, for a male-only initial release with late-acting bi-sex lethality. 


\section{Discussion}

We have theoretically investigated the use of underdominance as a method of vector population and disease suppression, using coupled models of mosquito ecology, genetics and epidemiology. We show that ecological barriers, that may manifest as decreased mating competitiveness or an increased rate of adult mortality, are the most important factors in determining the success chances of a control attempt (figs. $6 \mathrm{a}$ and 7). Low fitness of the engineered homozygotes (through 'leaky' toxicity from underdominant alleles, for example) can lead to high underdominance thresholds (fig. 4b) and hence could be economically costly (although it should be possible to engineer underdominant transgenes with a low ambient fitness cost). The efficiency of heterozygote lethality via toxin expression is less important (fig. 4a).

Previous investigations of underdominance drives as vector control only considered bi-sex releases. However, it may be hard to justify the release of biting insects and potential disease vectors. Our results suggest that population suppression can be achieved by releasing males carrying two homologous engineered underdominance constructs (though with a substantial increase in the release frequency threshold required to trigger the underdominance drive). Another partial solution to the problems associated with bi-sex releases (and one that we do not investigate here) is the proposal to link disease refractory genes to the engineered underdominance constructs, thus reducing the vectorial capacity of GM females $[8,58]$. For mosquito control, male-only releases are more plausible from a regulatory standpoint, so their increased threshold requirements (fig. 3) need to be accounted for.

Once an underdominant construct is released, population suppression occurs during the transition towards the new stable state. If this transitional period is in fact the gradual elimination of the engineered construct (i.e. the initial allele frequency was below that required to trigger the underdominant drive in the favour of the engineered construct), the temporary population suppression may be large and have tangible socioeconomic benefits (as noted by Serebrovsky [20], who theorised that pest populations controlled with temporary translocation strains would be "dealt a severe blow"). The population suppression resulting from a successful underdominance drive using the homologous system is so great that it may push the vector population below the entomological threshold necessary to transmit disease. For example, fig. 3b shows a situation where the homologous system control effort reduces the total vector population to less than $1 \%$ of the wild-type equilibrium, corresponding to $k=0.02$ vectors per host-lower than the $k=0.05$ boundary that a simple $R_{0}$ analysis of the disease model (2.9) gives as the minimum number of vectors per host required for the disease to spread. Similarly large population suppression was observed by Vanderplank [59,60] in field trials of tsetse fly control by underdominanttype methods (utilising the discovery that cross-breeds of two tsetse species exhibited reduced fecundity; the field trial results are published in [61]). Thus it should be possible to use certain underdominance systems as disease suppression technologies without the need to link anti-pathogen effector genes to the underdominant constructs.

Population eradication may not be the most beneficial result of a control programme due to the 
possibility of secondary vectors invading the abandoned niche [62]. This is certainly a possibility in some regions of the Americas where Ae. aegypti and Ae. albopictus live in close proximity and actively compete $[63,64]$. Approximating the minimum size of a primary vector population such that it could successfully occupy an ecological niche at the expense of a secondary vector would be an interesting problem for those aiming to implement genetic vector control technologies. Population replacement, rather than population suppression, may be the answer to such concerns (where the new population is genetically engineered to have a lower vectorial capacity), but is not without its own risks (as discussed above). The two non-homologous underdominance systems tested here show potential as agents of population replacement, with the amount of population suppression resulting from a successful underdominance gene drive depending on the value of the ambient transgene fitness cost, which governs the relative fitness of the engineered homozygotes (fig. 3).

Female-specific lethality acts to push underdominance thresholds higher (fig. 5a), suggesting that a greater initial investment is required. However, release attempts that fail to trigger the underdominance drive perform better (i.e. avert more infections) with female-specific, rather than bi-sex, lethality (fig. 5b). Due to the high threshold frequencies of male-only releases (for the homologous system in particular) and the possible difficulties in encouraging released mosquitoes to disseminate from the release site $[65,66]$ and assimilate into the wild breeding population [67,68], it may be a common occurrence during a control programme for a drive threshold not to be reached with a single bulk release. In this case, the economic and social benefit of averting a higher percentage of infections may outweigh the extra expense, in the form of extra releases, required to subsequently push a female-specific lethal release over the drive threshold compared with an bi-sex lethal release.

The mating success of the engineered homozygotes and heterozygotes with wild-type females is vitally important. Even a modest degree of sexual selection could generate strong behavioural resistance to the gene drive. This could take the form of mate choice by wild females or mating competition on the part of the wild males and could render large, expensive releases useless by increasing the underdominance threshold higher than had been accounted for (fig. 6a). In the control of species other than mosquitoes, behavioural resistance has been observed within a few generations $[69,70]$ and has resulted in control programmes being abandoned [71]. There are many reports of mosquito control field trials producing poor results due to reduced mating competitiveness of transgenic mosquitoes [13,68], and in most cases it is apparent that the ability to compete successfully for mates in laboratory conditions does not transfer perfectly to the field $[56,57,67,72,73]$. However, recent trials of self-limiting male-releases have shown more promise $[74,75]$. To determine how problematic behavioural resistance could be for the successful establishment and persistence of underdominance drives, and gene drives more generally, an important parameter to understand is the degree to which male mating success is determined by female choice. Variance in male quality has been the focus of mating behaviour research, whereas the role of female choice has largely been ignored. There is a need, then, for behavioural experiments aimed at providing insights into the importance of female mating preference and the speed with which preference can develop 
or change under strong selection pressure.

Artificial rearing and genetic engineering can lead to other barriers to success for an underdominance control programme by affecting the life history parameters of the transgenic insects. Field trials of transgenic mosquitoes have shown evidence of increased mortality in adults and larvae, longer development times to reach adulthood, lower fertility and a reduced range of dispersal $[55-57,65,66]$. We identify adult longevity as an important attribute for allowing a threshold drive to succeed (fig. 7) as a longer reproductive window gives the greatest chance that desirable genes will spread. Larval maturation rate and the strength and scale of density-dependent competition pressure are shown to have a lesser effect, independently, on the underdominance threshold. However, a lesser ability to compete for resources at the larval stage may lead to a lower maturation rate, and smaller, less developed larvae may emerge from pupation as stunted adults doomed to a shortened life (and with a lesser ability to compete for mates). Thus, the problems of ecological fitness costs manifested as altered life history parameters are likely to be compounded and attempts to establish an engineered population capable of triggering the underdominance threshold drive may be compromised. Indeed, field trials of translocation-bearing insects have reported many disadvantages compared with wild types [73]. Engineered underdominance systems may impose a lesser genetic load on individuals than translocations, but this type of gene drive system has thus far only been engineered in Drosophila [5, 26], and no underdominance system has yet been engineered that is capable of carrying a payload gene in an organism of medical or agricultural importance [8].

Future modelling work is required to investigate more fully the ramifications of our findings. For instance, capturing the evolution of mate choice as a dynamic process inside an ecological model would help determine whether behavioural resistance would develop quickly enough to prevent significant population suppression. Also, spatially explicit dynamics are vital for predicting the spread or containment of a control effort - understanding how threshold drives perform when movement between subpopulations occurs is necessary in judging their safety and viability. While previous studies have shown that small amounts of migration between a target and non-target population does not endanger the non-target population when an underdominance drive is used (in contrast to a homing gene drive), these models used pure population genetics approaches $[6,28]$. Ecological or environmental stochasticity might plausibly lead an underdominance threshold to be triggered by migration into a non-target population. It is clear, though, that understanding and accounting for the ecological and evolutionary effects - be they decreased adult longevity or the development of behavioural resistance via sexual selection - of any gene drive technology is vital if they are to be used successfully for mosquito vector control.

\section{Competing interests}

We have no competing interests.

\section{Authors' contributions}

DK, CEM, KK and MBB conceived the study. DK performed the mathematical analysis and drafted the manuscript. CEM, KK and MBB helped draft the manuscript. All authors gave final approval for 
publication.

Acknowledgments

The authors are grateful for financial support from DARPA.

References

[1] S. Bhatt, P. W. Gething, O. J. Brady, J. P. Messina, A. W. Farlow, C. L. Moyes, J. M. Drake, J. S. Brownstein, A. G. Hoen, O. Sankoh, M. F. Myers, D. B. George, T. Jaenisch, G. R. W. Wint, C. P. Simmons, T. W. Scott, J. J. Farrar, S. I. Hay, The global distribution and burden of dengue, Nature 496 (7446) (2013) 504-507.

[2] WHO, World Malaria Report 2016, World Health Organization, Geneva, 2016.

[3] WHO, Global Vector Control Response 2017-2030, World Health Organization, Geneva, 2017.

[4] D. Doolittle, Population genetics: basic principles, Advanced series in agricultural sciences, SpringerVerlag, Heidelberg, 1987.

[5] R. G. Reeves, J. Bryk, P. M. Altrock, J. A. Denton, F. A. Reed, First steps towards underdominant genetic transformation of insect populations, PLOS ONE 9 (5) (2014) 1-9. doi:10.1371/journal. pone. 0097557.

[6] P. M. Altrock, A. Traulsen, R. G. Reeves, F. A. Reed, Using underdominance to bi-stably transform local populations, Journal of Theoretical Biology 267 (1) (2010) 62-75. doi:http://dx.doi.org/ $10.1016 / j \cdot j t b i .2010 .08 .004$.

[7] P. M. Altrock, A. Traulsen, F. A. Reed, Stability properties of underdominance in finite subdivided populations, PLOS Computational Biology 7 (11) (2011) 1-10. doi:10.1371/journal.pcbi. 1002260.

[8] J. Champer, A. Buchman, O. S. Akbari, Cheating evolution: engineering gene drives to manipulate the fate of wild populations, Nat Rev Genet 17 (3) (2016) 146-159.

[9] D. Gurwitz, Gene drives raise dual-use concerns, Science 345 (6200) (2014) 1010-1010. doi:10. 1126/science.345.6200.1010-b.

[10] K. M. Esvelt, A. L. Smidler, F. Catteruccia, G. M. Church, Emerging technology: Concerning rnaguided gene drives for the alteration of wild populations, eLife 3 (2014) e03401. doi:10.7554/ eLife. 03401

[11] K. A. Oye, K. Esvelt, E. Appleton, F. Catteruccia, G. Church, T. Kuiken, S. B.-Y. Lightfoot, J. McNamara, A. Smidler, J. P. Collins, Regulating gene drives, Science 345 (6197) (2014) 626-628. doi:10.1126/science.1254287.

[12] G. D. Snell, An analysis of translocations in the mouse., Genetics 31 (1946) 157-180. 
[13] C. F. Curtis, T. Adak, Population replacement in culex fatigans by means of cytoplasmic incompatibility: 1. laboratory experiments with non-overlapping generations, Bulletin of the World Health Organization 51 (3) (1974) 249-255.

[14] P. T. McDonald, K. S. Rai, Population control potential of heterozygous translocations as determined by computer simulations, Bull. World Health Organ. 44 (6) (1971) 829-845.

[15] D. K. UPPAL, C. F. CURTIS, K. S. RAI, A double translocation heterozygote in Aedes aegypti, Journal of Communicable Diseases 6 (2) (1974) 98-101.

[16] N. Lorimer, L. P. Lounibos, J. L. Petersen, Field trials with a translocation homozygote in aedes aegypti for population replacement 1, Journal of Economic Entomology 69 (3) (1976) 405-409. doi:10.1093/jee/69.3.405.

[17] C. F. Curtis, Male-linked translocations and the control of insect pest populations, Experientia 31 (10) (1975) 1139-1141.

[18] A. S. ROBINSON, Progress in the use of chromosomal translocations for the control of insect pests, Biological Reviews 51 (1) (1976) 1-24. doi:10.1111/j.1469-185x.1976.tb01118.x.

[19] P. T. McDonald, W. Hausermann, N. Lorimer, Sterility introduced by release of genetically altered males to a domestic population of Aedes aegypti at the Kenya coast, Am J Trop Med Hyg 26 (3) (1977) 553-561.

[20] A. S. Serebrovsky, On the possibility of a new method for the control of insect pests, Zool. Zhurnal 19 (1940) 618-631.

[21] H. Laven, Eradicating mosquitoes using translocations, Nature 221 (5184) (1969) 958-959.

[22] H. Laven, J. Cousserans, G. Guille, Eradicating mosquitoes using translocations: a first field experiment, Nature 236 (5348) (1972) 456-457.

[23] G. G. Foster, M. J. Whitten, T. Prout, R. Gill, Chromosome rearrangements for the control of insect pests, Science 176 (4037) (1972) 875-880. doi:10.1126/science.176.4037.875.

[24] F. Gould, P. Schliekelman, Population genetics of autocidal control and strain replacement, Annu. Rev. Entomol. 49 (2004) 193-217.

[25] S. Davis, N. Bax, P. Grewe, Engineered underdominance allows efficient and economical introgression of traits into pest populations, Journal of Theoretical Biology 212 (1) (2001) 83-98. doi:http: //dx.doi.org/10.1006/jtbi.2001.2357.

[26] O. S. Akbari, K. D. Matzen, J. M. Marshall, H. Huang, C. M. Ward, B. A. Hay, A synthetic gene drive system for local, reversible modification and suppression of insect populations, Current Biology 23 (8) (2013) 671-677. doi:10.1016/j.cub.2013.02.059. 
[27] K. Magori, F. Gould, Genetically engineered underdominance for manipulation of pest populations: A deterministic model, Genetics 172 (4) (2006) 2613-2620. doi:10.1534/genetics.105.051789.

[28] C. S. Gokhale, R. G. Reeves, F. A. Reed, Dynamics of a combined medea-underdominant population transformation system, BMC Evolutionary Biology 14 (1) (2014) 98. doi:10.1186/ 1471-2148-14-98.

[29] M. P. Edgington, L. S. Alphey, Conditions for success of engineered underdominance gene drive systems, Journal of Theoretical Biology 430 (2017) 128-140. doi:http://dx.doi.org/10.1016/j. jtbi.2017.07.014.

[30] T. S. Bellows, The descriptive properties of some models for density dependence, Journal of Animal Ecology 50 (1) (1981) 139-156.

[31] J. L. Aron, R. M. May, The population dynamics of malaria, in: R. M. Anderson (Ed.), Population dynamics of infectious diseases: Theory and application, Population and Community Biology, Chapman and Hall, London, 1982, pp. 139-179.

[32] C. Dye, Models for the population dynamics of the yellow fever mosquito, aedes aegypti, Journal of Animal Ecology 53 (1) (1984) 247-268.

[33] T. R. E. Southwood, G. Murdie, M. Yasuno, R. J. Tonn, P. M. Reader, Studies on the life budget of Aedes aegypti in Wat Samphaya, Bangkok, Thailand, Bulletin of the World Health Organization 46 (2) (1972) 211-226.

[34] P. M. Sheppard, W. W. Macdonald, R. J. Tonn, B. Grab, The dynamics of an adult population of Aedes aegypti in relation to dengue haemorrhagic fever in bangkok, Journal of Animal Ecology 38 (3) (1969) 661-702.

[35] M. B. Hoshen, A. P. Morse, A weather-driven model of malaria transmission, Malaria Journal 3 (1) (2004) 32. doi:10.1186/1475-2875-3-32.

[36] V. Ermert, A. H. Fink, A. E. Jones, A. P. Morse, Development of a new version of the liverpool malaria model. i. refining the parameter settings and mathematical formulation of basic processes based on a literature review, Malaria Journal 10 (1) (2011) 35. doi:10.1186/1475-2875-10-35.

[37] V. Ermert, A. H. Fink, A. E. Jones, A. P. Morse, Development of a new version of the Liverpool Malaria Model. II. Calibration and validation for West Africa, Malaria Journal 10 (1) (2011) 62. doi:10.1186/1475-2875-10-62.

[38] A. Deredec, H. C. J. Godfray, A. Burt, Requirements for effective malaria control with homing endonuclease genes, Proceedings of the National Academy of Sciences 108 (43) (2011) E874-E880. doi:10.1073/pnas.1110717108 
[39] L. Molineaux, G. Gramiccia, The Garki project : research on the epidemiology and control of malaria in the Sudan savanna of West Africa, World Health Organization, Geneva, 1980.

[40] A. N. Clements, G. D. Paterson, The analysis of mortality and survival rates in wild populations of mosquitoes, Journal of Applied Ecology 18 (2) (1981) 373-399.

[41] K. N.-E. Jannat, B. D. Roitberg, Effects of larval density and feeding rates on larval life history traits in Anopheles gambiae s.s. (diptera: Culicidae), Journal of Vector Ecology 38 (1) (2013) 120-126.

[42] V. A. Alegana, S. P. Kigozi, J. Nankabirwa, E. Arinaitwe, R. Kigozi, H. Mawejje, M. Kilama, N. W. Ruktanonchai, C. W. Ruktanonchai, C. Drakeley, S. W. Lindsay, B. Greenhouse, M. R. Kamya, D. L. Smith, P. M. Atkinson, G. Dorsey, A. J. Tatem, Spatio-temporal analysis of malaria vector density from baseline through intervention in a high transmission setting, Parasites \& Vectors 9 (1) (2016) 637. doi:10.1186/s13071-016-1917-3.

[43] J.-F. Trape, E. Lefebvre-Zante, F. Legros, G. Ndiaye, H. Bouganali, P. Druilhe, G. Salem, Vector density gradients and the epidemiology of urban malaria in dakar, senegal, The American Journal of Tropical Medicine and Hygiene 47 (2) (1992) 181-189. doi:https://doi.org/10.4269/ajtmh. 1992.47 .181

[44] E. N. Ototo, J. P. Mbugi, C. L. Wanjala, G. Zhou, A. K. Githeko, G. Yan, Surveillance of malaria vector population density and biting behaviour in western kenya, Malaria Journal 14 (1) (2015) 244. doi:10.1186/s12936-015-0763-7.

[45] M. Kilama, D. L. Smith, R. Hutchinson, R. Kigozi, A. Yeka, G. Lavoy, M. R. Kamya, S. G. Staedke, M. J. Donnelly, C. Drakeley, B. Greenhouse, G. Dorsey, S. W. Lindsay, Estimating the annual entomological inoculation rate for plasmodium falciparum transmitted by anopheles gambiae s.l. using three sampling methods in three sites in uganda, Malaria Journal 13 (1) (2014) 111. doi: 10.1186/1475-2875-13-111.

[46] N. Windbichler, P. A. Papathanos, A. Crisanti, Targeting the $\mathrm{x}$ chromosome during spermatogenesis induces y chromosome transmission ratio distortion and early dominant embryo lethality in anopheles gambiae, PLOS Genetics 4 (12) (2008) 1-9. doi:10.1371/journal.pgen.1000291.

[47] R. Galizi, L. A. Doyle, M. Menichelli, F. Bernardini, A. Deredec, A. Burt, B. L. Stoddard, N. Windbichler, A. Crisanti, A synthetic sex ratio distortion system for the control of the human malaria mosquito, Nature Communications 5 (2014) 3977.

[48] P. Schliekelman, F. Gould, Pest control by the release of insects carrying a female-killing allele on multiple loci, J Econ Entomol 93 (6) (2000) 1566-1579.

[49] G. Fu, K. C. Condon, M. J. Epton, P. Gong, L. Jin, G. C. Condon, N. I. Morrison, T. H. Dafa'alla, L. Alphey, Female-specific insect lethality engineered using alternative splicing, Nat Biotech 25 (3) (2007) 353-357. 
[50] L. Jin, A. S. Walker, G. Fu, T. Harvey-Samuel, T. Dafa'alla, A. Miles, T. Marubbi, D. Granville, N. Humphrey-Jones, S. O’Connell, N. I. Morrison, L. Alphey, Engineered female-specific lethality for control of pest lepidoptera, ACS Synthetic Biology 2 (3) (2013) 160-166. doi:10.1021/sb300123m.

[51] G. Fu, R. S. Lees, D. Nimmo, D. Aw, L. Jin, P. Gray, T. U. Berendonk, H. White-Cooper, S. Scaife, H. Kim Phuc, O. Marinotti, N. Jasinskiene, A. A. James, L. Alphey, Female-specific flightless phenotype for mosquito control, Proceedings of the National Academy of Sciences 107 (10) (2010) 4550-4554. doi:10.1073/pnas.1000251107.

[52] G. M. C. Labbé, S. Scaife, S. A. Morgan, Z. H. Curtis, L. Alphey, Female-specific flightless (fsridl) phenotype for control of aedes albopictus, PLOS Neglected Tropical Diseases 6 (7) (2012) 1-8. doi:10.1371/journal.pntd.0001724.

[53] N. Alphey, P. G. Coleman, C. A. Donnelly, L. Alphey, Managing insecticide resistance by mass release of engineered insects, J Econ Entomol 100 (5) (2007) 1642-1649.

[54] N. Alphey, A. L. Bonsall, M. B., Combining pest control and resistance management: synergy of engineered insects with bt crops, J Econ Entomol 102 (2) (2009) 717-732.

[55] J. A. Seawright, P. E. Kaiser, N. L. Willis, D. A. Dame, Field competitiveness of double translocation heterozygote males of aedes aegypti (1.), Journal of Medical Entomology 13 (2) (1976) 208. doi: 10.1093/jmedent/13.2.208.

[56] H. M. Ferguson, B. John, K. Ng'habi, B. G. J. Knols, Redressing the sex imbalance in knowledge of vector biology, Trends in Ecology \& Evolution 20 (4) (2005) 202-209. doi:http://dx.doi.org/ $10.1016 / j . t r e e .2005 .02 .003$.

[57] M. Q. Benedict, A. S. Robinson, The first releases of transgenic mosquitoes: an argument for the sterile insect technique, Trends in Parasitology 19 (8) (2003) 349-355. doi:http://dx.doi.org/ $10.1016 / \mathrm{S} 1471-4922(03) 00144-2$.

[58] S. P. Sinkins, F. Gould, Gene drive systems for insect disease vectors, Nat Rev Genet 7 (6) (2006) 427-435.

[59] F. L. Vanderplank, Hybridization between glossina species and suggested new method for control of certain species of tsetse, Nature 154 (1944) 607-608.

[60] F. L. Vanderplank, Experiments in the hybridisation of tsetse-flies (glossina, diptera) and the possibility of a new method of control, Transactions of the Royal Entomological Society of London 98 (1) (1947) 1-18. doi:10.1111/j.1365-2311.1947.tb01049.x.

[61] W. Klassen, C. F. Curtis, History of the sterile insect technique, in: V. A. Dyck, J. Hendrichs, A. S. Robinson (Eds.), Sterile Insect Technique: Principles and Practice in Area-Wide Integrated Pest Management, Springer Netherlands, Dordrecht, 2005, pp. 209-232. 
[62] M. B. Bonsall, L. Yakob, N. Alphey, L. Alphey, Transgenic control of vectors: the effects of interspecific interactions, Isr. J. Ecol. Evol. 56 (2010) 353-370.

[63] M. A. H. Braks, N. A. Honório, L. P. Lounibos, R. Lourenço-De-Oliveira, S. A. Juliano, Interspecific competition between two invasive species of container mosquitoes, aedes aegypti and aedes albopictus (diptera: Culicidae), in brazil, Annals of the Entomological Society of America 97 (1) (2004) 130-139.

[64] L. P. Lounibos, I. Bargielowski, M. C. Carrasquilla, N. Nishimura, Coexistence of aedes aegypti and aedes albopictus (diptera: Culicidae) in peninsular florida two decades after competitive displacements, Journal of Medical Entomology 53 (6) (2016) 1385. doi:10.1093/jme/tjw122.

[65] R. Lacroix, A. R. McKemey, N. Raduan, L. Kwee Wee, W. Hong Ming, T. Guat Ney, S. Rahidah A.A., S. Salman, S. Subramaniam, O. Nordin, N. Hanum A.T., C. Angamuthu, S. Marlina Mansor, R. S. Lees, N. Naish, S. Scaife, P. Gray, G. Labbé, C. Beech, D. Nimmo, L. Alphey, S. S. Vasan, L. Han Lim, N. Wasi A., S. Murad, Open field release of genetically engineered sterile male aedes aegypti in malaysia, PLOS ONE 7 (8) (2012) 1-9. doi:10.1371/journal.pone.0042771.

[66] P. Winskill, D. O. Carvalho, M. L. Capurro, L. Alphey, C. A. Donnelly, A. R. McKemey, Dispersal of engineered male aedes aegypti mosquitoes, PLOS Neglected Tropical Diseases 9 (11) (2015) 1-18. doi:10.1371/journal.pntd.0004156

[67] W. K. Reisen, R. H. Baker, R. K. Sakai, F. Mahmood, H. R. Rathor, K. Raana, G. Toqir, Anopheles culicifacies giles:1 mating behavior and competitiveness in nature of chemosterilized males carrying a genetic sexing system, Annals of the Entomological Society of America 74 (4) (1981) 395. doi: 10.1093/aesa/74.4.395

[68] W. K. Reisen, Lessons from the past: historical studies by the university of maryland and the university of california, berkeley, in: W. Takken, T. W. Scott (Eds.), Ecological aspects for application of genetically modified mosquitoes, no. 2 in Frontis Series, Kluwer Academic Publishers, Dordrecht, 2003, pp. 25-32.

[69] R. C. Bushland, Letter: Screwworm eradication program, Science 184 (4140) (1974) 1010-1011.

[70] Y. Hibino, O. Iwahashi, Appearance of wild females unreceptive to sterilized males on Okinawa Is. in the eradication program of the melon fly, dacus cucurbitae COQUILLETT (Diptera: Tephritidae), Applied Entomology and Zoology 26 (2) (1991) 265-270. doi:10.1303/aez.26.265.

[71] D. O. McInnis, D. R. Lance, C. G. Jackson, Behavioral resistance to the sterile insect technique by mediterranean fruit fly (Diptera: Tephritidae) in Hawaii, Ann Entomol Soc Am 89 (1996) 739-744. doi:10.1303/aez.26.265

[72] S. M. Asman, P. T. McDonald, T. Prout, Field studies of genetic control systems for mosquitoes, Annu Rev Entomol 26 (1981) 289-318. doi:10.1146/annurev.en.26.010181.001445. 
[73] L. P. Lounibos, Genetic-control trials and the ecology of aedes aegypti at the kenya coast, in: W. Takken, T. W. Scott (Eds.), Ecological aspects for application of genetically modified mosquitoes, no. 2 in Frontis Series, Kluwer Academic Publishers, Dordrecht, 2003, pp. 33-43.

[74] A. F. Harris, D. Nimmo, A. R. McKemey, N. Kelly, S. Scaife, C. A. Donnelly, C. Beech, W. D. Petrie, L. Alphey, Field performance of engineered male mosquitoes, Nat Biotech 29 (11) (2011) 1034-1037.

[75] D. O. Carvalho, A. R. McKemey, L. Garziera, R. Lacroix, C. A. Donnelly, L. Alphey, A. Malavasi, M. L. Capurro, Suppression of a field population of aedes aegypti in brazil by sustained release of transgenic male mosquitoes, PLOS Neglected Tropical Diseases 9 (7) (2015) 1-15. doi:10.1371/ journal.pntd.0003864.

[76] A. M. Hasofer, A continuous-time model in population genetics, Journal of Theoretical Biology 11 (1966) 150-163

\section{Appendix}

\section{A. Genotypic offspring proportions}

\section{A.1. Random mating}

Working through the population genetic matings for the homologous underdominance system leads to the following proportions of each sex in each genotype at any given time:

$p_{1}=\frac{1}{2 N_{m} N_{f}}\left(N_{1} \hat{N}_{1}+\frac{1}{2}\left(N_{1} \hat{N}_{2}+\hat{N}_{1} N_{2}\right)+\frac{1}{2}\left(N_{1} \hat{N}_{3}+\hat{N}_{1} N_{3}\right)+\frac{1}{4} N_{2} \hat{N}_{2}+\frac{1}{4} N_{3} \hat{N}_{3}+\frac{1}{4}\left(N_{2} \hat{N}_{3}+\hat{N}_{2} N_{3}\right)\right)$,

$p_{2}=\frac{1}{2 N_{m} N_{f}}\left(\frac{1}{2}\left(N_{1} \hat{N}_{2}+\hat{N}_{1} N_{2}\right)+\frac{1}{2}\left(\hat{N}_{1} N_{4}+N_{1} \hat{N}_{4}\right)+\left(\hat{N}_{1} N_{5}+N_{1} \hat{N}_{5}\right)+\frac{1}{2} N_{2} \hat{N}_{2}+\frac{1}{4}\left(N_{2} \hat{N}_{3}+\hat{N}_{2} N_{3}\right)\right.$ $\left.+\frac{1}{4}\left(\hat{N}_{2} N_{4}+N_{2} \hat{N}_{4}\right)+\frac{1}{2}\left(\hat{N}_{2} N_{5}+N_{2} \hat{N}_{5}\right)+\frac{1}{2}\left(N_{3} \hat{N}_{5}+\hat{N}_{3} N_{5}\right)+\frac{1}{4}\left(\hat{N}_{3} N_{4}+N_{3} \hat{N}_{4}\right)\right)$,

$p_{3}=\frac{1}{2 N_{m} N_{f}}\left(\frac{1}{2}\left(N_{1} \hat{N}_{3}+\hat{N}_{1} N_{3}\right)+\frac{1}{2}\left(\hat{N}_{1} N_{4}+N_{1} \hat{N}_{4}\right)+\left(N_{1} \hat{N}_{6}+\hat{N}_{1} N_{6}\right)+\frac{1}{2}\left(N_{2} \hat{N}_{6}+\hat{N}_{2} N_{6}\right)\right.$ $\left.+\frac{1}{4}\left(N_{2} \hat{N}_{3}+\hat{N}_{2} N_{3}\right)+\frac{1}{4}\left(\hat{N}_{2} N_{4}+N_{2} \hat{N}_{4}\right)+\frac{1}{2} N_{3} \hat{N}_{3}+\frac{1}{2}\left(N_{3} \hat{N}_{6}+\hat{N}_{3} N_{6}\right)+\frac{1}{4}\left(\hat{N}_{3} N_{4}+N_{3} \hat{N}_{4}\right)\right)$,

$$
\begin{aligned}
p_{4}= & \frac{1}{2 N_{m} N_{f}}\left(\frac{1}{4}\left(N_{2} \hat{N}_{3}+\hat{N}_{2} N_{3}\right)+\frac{1}{4}\left(\hat{N}_{2} N_{4}+N_{2} \hat{N}_{4}\right)+\frac{1}{2}\left(N_{2} \hat{N}_{6}+\hat{N}_{2} N_{6}\right)+\frac{1}{4}\left(\hat{N}_{3} N_{4}+N_{3} \hat{N}_{4}\right)\right. \\
& \left.+\frac{1}{2}\left(N_{3} \hat{N}_{5}+\hat{N}_{3} N_{5}\right)+\left(N_{5} \hat{N}_{6}+\hat{N}_{5} N_{6}\right)+\frac{1}{2}\left(\hat{N}_{5} N_{4}+N_{5} \hat{N}_{4}\right)+\frac{1}{2}\left(\hat{N}_{6} N_{4}+N_{6} \hat{N}_{4}\right)+\frac{1}{2} N_{4} \hat{N}_{4}\right),
\end{aligned}
$$

${ }^{596} \quad p_{5}=\frac{1}{2 N_{m} N_{f}}\left(\frac{1}{4} N_{2} \hat{N}_{2}+\frac{1}{4}\left(\hat{N}_{2} N_{4}+N_{2} \hat{N}_{4}\right)+\frac{1}{2}\left(N_{2} \hat{N}_{5}+\hat{N}_{2} N_{5}\right)+\frac{1}{4} N_{4} \hat{N}_{4}+\frac{1}{2}\left(\hat{N}_{5} N_{4}+N_{5} \hat{N}_{4}\right)+N_{5} \hat{N}_{5}\right)$,

$$
p_{6}=\frac{1}{2 N_{m} N_{f}}\left(\frac{1}{4} N_{3} \hat{N}_{3}+\frac{1}{4}\left(\hat{N}_{3} N_{4}+N_{3} \hat{N}_{4}\right)+\frac{1}{2}\left(N_{3} \hat{N}_{6}+\hat{N}_{3} N_{6}\right)+\frac{1}{4} N_{4} \hat{N}_{4}+\frac{1}{2}\left(\hat{N}_{6} N_{4}+N_{6} \hat{N}_{4}\right)+N_{6} \hat{N}_{6}\right),
$$


where $p_{1}, p_{2}, p_{3}, p_{4}, p_{5}$ and $p_{6}$ relate to genotypes $A A, A \alpha, A \beta, \alpha \beta, \alpha \alpha$ and $\beta \beta$ respectively. Each genotype is split explicitly into males $\left(\hat{N}_{i}\right)$ and females $\left(N_{i}\right)$, though gender imbalance may only occur through female-specific lethality or in the $\alpha \beta$ genotype through releases of male mosquitoes (i.e. offspring are generated with a $1: 1$ sex ratio, giving the factor of $1 / 2$ outside the brackets in each $p_{i}$ definition).

For the non-homologous underdominance system, the offspring proportions of each sex in each genotype are given by

$$
\begin{aligned}
p_{1}= & \frac{1}{2 N_{m} N_{f}}\left(N_{1} \hat{N}_{1}+\frac{1}{2}\left(N_{1} \hat{N}_{2}+\hat{N}_{1} N_{2}\right)+\frac{1}{2}\left(N_{1} \hat{N}_{3}+\hat{N}_{1} N_{3}\right)+\frac{1}{4}\left(N_{1} \hat{N}_{4}+\hat{N}_{1} N_{4}\right)+\frac{1}{4} N_{2} \hat{N}_{2}\right. \\
& \left.+\frac{1}{4}\left(N_{2} \hat{N}_{3}+\hat{N}_{2} N_{3}\right)+\frac{1}{8}\left(N_{2} \hat{N}_{4}+\hat{N}_{2} N_{4}\right)+\frac{1}{4} N_{3} \hat{N}_{3}+\frac{1}{8}\left(N_{3} \hat{N}_{4}+\hat{N}_{3} N_{4}\right)+\frac{1}{16} N_{4} \hat{N}_{4}\right), \\
p_{2}= & \frac{1}{2 N_{m} N_{f}}\left(\frac{1}{2}\left(N_{1} \hat{N}_{2}+\hat{N}_{1} N_{2}\right)+\frac{1}{4}\left(N_{1} \hat{N}_{4}+\hat{N}_{1} N_{4}\right)+\left(N_{1} \hat{N}_{5}+\hat{N}_{1} N_{5}\right)+\frac{1}{2}\left(N_{1} \hat{N}_{7}+\hat{N}_{1} N_{7}\right)\right. \\
& +\frac{1}{2} N_{2} \hat{N}_{2}+\frac{1}{4}\left(N_{2} \hat{N}_{3}+\hat{N}_{2} N_{3}\right)+\frac{1}{4}\left(N_{2} \hat{N}_{4}+\hat{N}_{2} N_{4}\right)+\frac{1}{2}\left(N_{2} \hat{N}_{5}+\hat{N}_{2} N_{5}\right)+\frac{1}{4}\left(N_{2} \hat{N}_{7}+\hat{N}_{2} N_{7}\right) \\
& +\frac{1}{8}\left(N_{3} \hat{N}_{4}+\hat{N}_{3} N_{4}\right)+\frac{1}{2}\left(N_{3} \hat{N}_{5}+\hat{N}_{3} N_{5}\right)+\frac{1}{4}\left(N_{3} \hat{N}_{7}+\hat{N}_{3} N_{7}\right)+\frac{1}{8} N_{4} \hat{N}_{4}+\frac{1}{4}\left(N_{4} \hat{N}_{5}+\hat{N}_{4} N_{5}\right) \\
& \left.+\frac{1}{8}\left(N_{4} \hat{N}_{7}+\hat{N}_{4} N_{7}\right)\right), \\
p_{3}= & \frac{1}{2 N_{m} N_{f}}\left(\frac{1}{2}\left(N_{1} \hat{N}_{3}+\hat{N}_{1} N_{3}\right)+\frac{1}{4}\left(N_{1} \hat{N}_{4}+\hat{N}_{1} N_{4}\right)+\left(N_{1} \hat{N}_{6}+\hat{N}_{1} N_{6}\right)+\frac{1}{2}\left(N_{1} \hat{N}_{8}+\hat{N}_{1} N_{8}\right)\right. \\
& +\frac{1}{2} N_{3} \hat{N}_{3}+\frac{1}{4}\left(N_{2} \hat{N}_{3}+\hat{N}_{2} N_{3}\right)+\frac{1}{8}\left(N_{2} \hat{N}_{4}+\hat{N}_{2} N_{4}\right)+\frac{1}{2}\left(N_{2} \hat{N}_{6}+\hat{N}_{2} N_{6}\right)+\frac{1}{4}\left(N_{2} \hat{N}_{8}+\hat{N}_{2} N_{8}\right) \\
& +\frac{1}{4}\left(N_{3} \hat{N}_{4}+\hat{N}_{3} N_{4}\right)+\frac{1}{2}\left(N_{3} \hat{N}_{6}+\hat{N}_{3} N_{6}\right)+\frac{1}{4}\left(N_{3} \hat{N}_{8}+\hat{N}_{3} N_{8}\right)+\frac{1}{8} N_{4} \hat{N}_{4}+\frac{1}{4}\left(N_{4} \hat{N}_{6}+\hat{N}_{4} N_{6}\right) \\
& \left.+\frac{1}{8}\left(N_{4} \hat{N}_{8}+\hat{N}_{4} N_{8}\right)\right),
\end{aligned}
$$

$$
\begin{aligned}
p_{4}= & \frac{1}{2 N_{m} N_{f}}\left(\frac{1}{4}\left(N_{1} \hat{N}_{4}+\hat{N}_{1} N_{4}\right)+\frac{1}{2}\left(N_{1} \hat{N}_{7}+\hat{N}_{1} N_{7}\right)+\frac{1}{2}\left(N_{1} \hat{N}_{8}+\hat{N}_{1} N_{8}\right)+\left(N_{1} \hat{N}_{9}+\hat{N}_{1} N_{9}\right)\right. \\
& +\frac{1}{4}\left(N_{2} \hat{N}_{3}+\hat{N}_{2} N_{3}\right)+\frac{1}{4}\left(N_{2} \hat{N}_{4}+\hat{N}_{2} N_{4}\right)+\frac{1}{2}\left(N_{2} \hat{N}_{6}+\hat{N}_{2} N_{6}\right)+\frac{1}{4}\left(N_{2} \hat{N}_{7}+\hat{N}_{2} N_{7}\right)
\end{aligned}
$$$$
+\frac{1}{2}\left(N_{2} \hat{N}_{8}+\hat{N}_{2} N_{8}\right)+\frac{1}{2}\left(N_{2} \hat{N}_{9}+\hat{N}_{2} N_{9}\right)+\frac{1}{4}\left(N_{3} \hat{N}_{4}+\hat{N}_{3} N_{4}\right)+\frac{1}{2}\left(N_{3} \hat{N}_{5}+\hat{N}_{3} N_{5}\right)
$$$$
+\frac{1}{2}\left(N_{3} \hat{N}_{7}+\hat{N}_{3} N_{7}\right)+\frac{1}{4}\left(N_{3} \hat{N}_{8}+\hat{N}_{3} N_{8}\right)+\frac{1}{2}\left(N_{3} \hat{N}_{9}+\hat{N}_{3} N_{9}\right)+\frac{1}{4} N_{4} \hat{N}_{4}+\frac{1}{4}\left(N_{4} \hat{N}_{5}+\hat{N}_{4} N_{5}\right)
$$$$
+\frac{1}{4}\left(N_{4} \hat{N}_{6}+\hat{N}_{4} N_{6}\right)+\frac{1}{4}\left(N_{4} \hat{N}_{7}+\hat{N}_{4} N_{7}\right)+\frac{1}{4}\left(N_{4} \hat{N}_{8}+\hat{N}_{4} N_{8}\right)+\frac{1}{4}\left(N_{4} \hat{N}_{9}+\hat{N}_{4} N_{9}\right)
$$

$$
\begin{aligned}
& \left.+\left(N_{5} \hat{N}_{6}+\hat{N}_{5} N_{6}\right)+\frac{1}{2}\left(N_{5} \hat{N}_{8}+\hat{N}_{5} N_{8}\right)+\frac{1}{2}\left(N_{6} \hat{N}_{7}+\hat{N}_{6} N_{7}\right)+\frac{1}{4}\left(N_{7} \hat{N}_{8}+\hat{N}_{7} N_{8}\right)\right), \\
p_{5}= & \frac{1}{2 N_{m} N_{f}}\left(\frac{1}{4} N_{2} \hat{N}_{2}+\frac{1}{8}\left(N_{2} \hat{N}_{4}+\hat{N}_{2} N_{4}\right)+\frac{1}{2}\left(N_{2} \hat{N}_{5}+\hat{N}_{2} N_{5}\right)+\frac{1}{4}\left(N_{2} \hat{N}_{7}+\hat{N}_{2} N_{7}\right)+\frac{1}{16} N_{4} \hat{N}_{4}\right.
\end{aligned}
$$$$
\left.+\frac{1}{4}\left(N_{4} \hat{N}_{5}+\hat{N}_{4} N_{5}\right)+\frac{1}{8}\left(N_{4} \hat{N}_{7}+\hat{N}_{4} N_{7}\right)+N_{5} \hat{N}_{5}+\frac{1}{2}\left(N_{5} \hat{N}_{7}+\hat{N}_{5} N_{7}\right)+\frac{1}{4} N_{7} \hat{N}_{7}\right),
$$

$$
\begin{aligned}
p_{6}= & \frac{1}{2 N_{m} N_{f}}\left(\frac{1}{4} N_{3} \hat{N}_{3}+\frac{1}{8}\left(N_{3} \hat{N}_{4}+\hat{N}_{3} N_{4}\right)+\frac{1}{2}\left(N_{3} \hat{N}_{6}+\hat{N}_{3} N_{6}\right)+\frac{1}{4}\left(N_{3} \hat{N}_{8}+\hat{N}_{3} N_{8}\right)+\frac{1}{16} N_{4} \hat{N}_{4}\right. \\
& \left.+\frac{1}{4}\left(N_{4} \hat{N}_{6}+\hat{N}_{4} N_{6}\right)+\frac{1}{8}\left(N_{4} \hat{N}_{8}+\hat{N}_{4} N_{8}\right)+N_{6} \hat{N}_{6}+\frac{1}{2}\left(N_{6} \hat{N}_{8}+\hat{N}_{6} N_{8}\right)+\frac{1}{4} N_{8} \hat{N}_{8}\right), \\
p_{7}= & \frac{1}{2 N_{m} N_{f}}\left(\frac{1}{8}\left(N_{2} \hat{N}_{4}+\hat{N}_{2} N_{4}\right)+\frac{1}{4}\left(N_{2} \hat{N}_{7}+\hat{N}_{2} N_{7}\right)+\frac{1}{4}\left(N_{2} \hat{N}_{8}+\hat{N}_{2} N_{8}\right)+\frac{1}{2}\left(N_{2} \hat{N}_{9}+\hat{N}_{2} N_{9}\right)\right. \\
& +\frac{1}{8} N_{4} \hat{N}_{4}+\frac{1}{4}\left(N_{4} \hat{N}_{5}+\hat{N}_{4} N_{5}\right)+\frac{1}{4}\left(N_{4} \hat{N}_{7}+\hat{N}_{4} N_{7}\right)+\frac{1}{8}\left(N_{4} \hat{N}_{8}+\hat{N}_{4} N_{8}\right)+\frac{1}{4}\left(N_{4} \hat{N}_{9}+\hat{N}_{4} N_{9}\right) \\
& +\frac{1}{2}\left(N_{5} \hat{N}_{7}+\hat{N}_{5} N_{7}\right)+\frac{1}{2}\left(N_{5} \hat{N}_{8}+\hat{N}_{5} N_{8}\right)+\left(N_{5} \hat{N}_{9}+\hat{N}_{5} N_{9}\right)+\frac{1}{2} N_{7} \hat{N}_{7}+\frac{1}{4}\left(N_{7} \hat{N}_{8}+\hat{N}_{7} N_{8}\right)
\end{aligned}
$$




$$
\begin{aligned}
& \left.+\frac{1}{2}\left(N_{7} \hat{N}_{9}+\hat{N}_{7} N_{9}\right)\right) \\
p_{8}= & \frac{1}{2 N_{m} N_{f}}\left(\frac{1}{8}\left(N_{3} \hat{N}_{4}+\hat{N}_{3} N_{4}\right)+\frac{1}{4}\left(N_{3} \hat{N}_{7}+\hat{N}_{3} N_{7}\right)+\frac{1}{4}\left(N_{3} \hat{N}_{8}+\hat{N}_{3} N_{8}\right)+\frac{1}{2}\left(N_{3} \hat{N}_{9}+\hat{N}_{3} N_{9}\right)\right. \\
& +\frac{1}{8} N_{4} \hat{N}_{4}+\frac{1}{4}\left(N_{4} \hat{N}_{6}+\hat{N}_{4} N_{6}\right)+\frac{1}{8}\left(N_{4} \hat{N}_{7}+\hat{N}_{4} N_{7}\right)+\frac{1}{4}\left(N_{4} \hat{N}_{8}+\hat{N}_{4} N_{8}\right)+\frac{1}{4}\left(N_{4} \hat{N}_{9}+\hat{N}_{4} N_{9}\right) \\
& +\frac{1}{2}\left(N_{6} \hat{N}_{7}+\hat{N}_{6} N_{7}\right)+\frac{1}{2}\left(N_{6} \hat{N}_{8}+\hat{N}_{6} N_{8}\right)+\left(N_{6} \hat{N}_{9}+\hat{N}_{6} N_{9}\right)+\frac{1}{2} N_{8} \hat{N}_{8}+\frac{1}{4}\left(N_{7} \hat{N}_{8}+\hat{N}_{7} N_{8}\right) \\
& \left.+\frac{1}{2}\left(N_{8} \hat{N}_{9}+\hat{N}_{8} N_{9}\right)\right), \\
p_{9}= & \frac{1}{2 N_{m} N_{f}}\left(\frac{1}{16} N_{4} \hat{N}_{4}+\frac{1}{8}\left(N_{4} \hat{N}_{7}+\hat{N}_{4} N_{7}\right)+\frac{1}{8}\left(N_{4} \hat{N}_{8}+\hat{N}_{4} N_{8}\right)+\frac{1}{4}\left(N_{4} \hat{N}_{9}+\hat{N}_{4} N_{9}\right)+\frac{1}{4} N_{7} \hat{N}_{7}\right. \\
& \left.+\frac{1}{4}\left(N_{7} \hat{N}_{8}+\hat{N}_{7} N_{8}\right)+\frac{1}{2}\left(N_{7} \hat{N}_{9}+\hat{N}_{7} N_{9}\right)+\frac{1}{4} N_{8} \hat{N}_{8}+\frac{1}{2}\left(N_{8} \hat{N}_{9}+\hat{N}_{8} N_{9}\right)+N_{9} \hat{N}_{9}\right),
\end{aligned}
$$

where $p_{1}, p_{2}, p_{3}, p_{4}, p_{5}, p_{6}, p_{7}, p_{8}$ and $p_{9}$ relate to genotypes $A A B B, A \alpha B B, A A B \beta, A \alpha B \beta, \alpha \alpha B B$, $A A \beta \beta, \alpha \alpha B \beta, A \alpha \beta \beta$ and $\alpha \alpha \beta \beta$, respectively.

\section{A.2. Wild-type mating preference}

Consider the simple case of extreme underdominance, where two alleles $A$ and $a$ form three genotypes $A A, A a$ and $a a$, and take $A A$ to be the wild type. Without mating preference the proportions $p_{i}$ of the next generation that belong to each genotype will be

$$
\begin{aligned}
p_{A A}= & \frac{1}{2 N_{m} N_{f}}\left(N_{A A} \hat{N}_{A A}+\frac{1}{2}\left(N_{A A} \hat{N}_{A a}+N_{A a} \hat{N}_{A A}\right)+\frac{1}{4} N_{A a} \hat{N}_{A a}\right), \\
p_{A a}= & \frac{1}{2 N_{m} N_{f}}\left(\frac{1}{2}\left(N_{A A} \hat{N}_{A a}+N_{A a} \hat{N}_{A A}\right)+N_{A A} \hat{N}_{a a}+N_{a a} \hat{N}_{A A}+\frac{1}{2} N_{A a} \hat{N}_{A a}\right. \\
& \left.\quad+\frac{1}{2}\left(N_{A a} \hat{N}_{a a}+N_{a a} \hat{N}_{A a}\right)\right), \\
p_{a a}= & \frac{1}{2 N_{m} N_{f}}\left(\frac{1}{4} N_{A a} \hat{N}_{A a}+\frac{1}{2}\left(N_{A a} \hat{N}_{a a}+N_{a a} \hat{N}_{A a}\right)+N_{a a} \hat{N}_{a a}\right) .
\end{aligned}
$$

We model mating preference in the wild-type female population by defining the parameters $\xi$ and $\chi$. A proportion $\xi$ of the wild-type females from each encounter with males who are not wild type will choose instead to mate with wild-type males. The number of resulting matings between wild-type males and females increases by a related proportion $\chi$. With mating preference we find

$$
\begin{aligned}
\bar{p}_{A A}= & \frac{1}{N_{m} N_{f}}\left((1+\chi) N_{A A} \hat{N}_{A A}+\frac{1}{2}\left((1-\xi) N_{A A} \hat{N}_{A a}+N_{A a} \hat{N}_{A A}\right)+\frac{1}{4} N_{A a} \hat{N}_{A a}\right), \\
\bar{p}_{A a}= & \frac{1}{N_{m} N_{f}}\left(\frac{1}{2}\left((1-\xi) N_{A A} \hat{N}_{A a}+N_{A a} \hat{N}_{A A}\right)+(1-\xi) N_{A A} \hat{N}_{a a}+N_{a a} \hat{N}_{A A}+\frac{1}{2} N_{A a} \hat{N}_{A a}\right. \\
& \left.\quad+\frac{1}{2}\left(N_{A a} \hat{N}_{a a}+N_{a a} \hat{N}_{A a}\right)\right), \\
\bar{p}_{a a}= & \frac{1}{N_{m} N_{f}}\left(\frac{1}{4} N_{A a} \hat{N}_{A a}+\frac{1}{2}\left(N_{A a} \hat{N}_{a a}+N_{a a} \hat{N}_{A a}\right)+N_{a a} \hat{N}_{a a}\right) .
\end{aligned}
$$

With wild-type mating preference, we expect $\bar{p}_{A A}>p_{A A}, \bar{p}_{A a}<p_{A a}$ and $\bar{p}_{a a}=p_{a a}$. The constraint $\sum_{i} p_{i}=1=\sum_{i} \bar{p}_{i}$ must also still hold. Taking these sums over the proportions and then subtracting one 
from the other we may find an expression for $\chi$ in terms of $\xi$ :

$$
\chi=\frac{\frac{1}{2} \hat{N}_{A a}+\frac{1}{2} \hat{N}_{A a}+\hat{N}_{a a}}{\hat{N}_{A A}} \xi,
$$

where the fraction is simply the sum of males in non-preferred matings over the number of males in the preferred mating. Thus we may use (A.5) to define the mating success parameter $\chi$, for a given $\xi$, in our engineered underdominance models with six and nine genotypes,

$$
\chi=\frac{\sum_{i=2}^{n_{g}} \hat{N}_{i}}{\hat{N}_{1}} \xi,
$$

where $n_{g}$ takes the value six and nine, respectively.

\section{B. Genotype fitness from transgene fitness costs}

Here we tabulate how the relative fitness of each genotype is constructed multiplicatively from the costs imposed by the constituent alleles of the genotype (with wild-type alleles imposing no fitness cost).

The non-homologous underdominance system is treated in tables B.4-B.7. The fitness table for the homologous underdominance system is repeated here for completeness, table B.8.

\begin{tabular}{lcccccc}
\hline & & \multicolumn{5}{c}{ Genotype } \\
Lethality & $A A B B$ & $A \alpha B B$ & $A \alpha B \beta$ & $\alpha \alpha B B$ & $\alpha \alpha B \beta$ & \\
& & $A A B \beta$ & & $A A \beta \beta$ & $A \alpha \beta \beta$ & $\alpha \beta \beta$ \\
\hline BSL & 1 & $\kappa_{\mathrm{a}} \kappa_{\ell}$ & $\kappa_{\mathrm{a}}^{2}$ & $\kappa_{\mathrm{a}}^{2} \kappa_{\ell}^{2}$ & $\kappa_{\mathrm{a}}^{3} \kappa_{\ell}$ & $\kappa_{\mathrm{a}}^{4}$ \\
FSL & 1 & $\kappa_{\mathrm{a}}$ & $\kappa_{\mathrm{a}}^{2}$ & $\kappa_{\mathrm{a}}^{2}$ & $\kappa_{\mathrm{a}}^{3}$ & $\kappa_{\mathrm{a}}^{4}$ \\
\hline
\end{tabular}

Table B.4: Male genotype fitness for the weakly-suppressed non-homologous underdominance system for bi-sex lethality (BSL) and female-specific lethality (FSL); $\kappa_{\mathrm{a}}=1-c_{\mathrm{a}}$ and $\kappa_{\ell}=1-c_{\ell}$ where $c_{\mathrm{a}}$ is the ambient fitness cost of carrying a single transgene and $c_{\ell}$ is the (partially) lethal fitness cost of carrying an unsuppressed toxin gene. Fitness costs are assumed to combine multiplicatively.

\begin{tabular}{lcccccc}
\hline \multicolumn{7}{c}{ Genotype } \\
Lethality & $A A B B$ & $A \alpha B B$ & \multirow{2}{*}{$A \alpha \beta$} & $\alpha \alpha B B$ & $\alpha \alpha B \beta$ & \\
& & $A A B \beta$ & & $A A \beta \beta$ & $A \alpha \beta \beta$ & $\alpha \alpha \beta$ \\
\hline BSL/FSL & 1 & $\kappa_{\mathrm{a}} \kappa_{\ell}$ & $\kappa_{\mathrm{a}}^{2}$ & $\kappa_{\mathrm{a}}^{2} \kappa_{\ell}^{2}$ & $\kappa_{\mathrm{a}}^{3} \kappa_{\ell}$ & $\kappa_{\mathrm{a}}^{4}$ \\
\hline
\end{tabular}

Table B.5: As in table B.4: female genotype fitness for weakly-suppressed non-homologous underdominance system.

\begin{tabular}{lcccccc}
\hline \multicolumn{7}{c}{ Genotype } \\
Lethality & $A A B B$ & $A \alpha B B$ & $A \alpha B \beta$ & $\alpha \alpha B B$ & $\alpha \alpha B \beta$ & \\
& & $A A B \beta$ & & $A A \beta \beta$ & $A \alpha \beta \beta$ & $\alpha \alpha \beta$ \\
\hline BSL & 1 & $\kappa_{\mathrm{a}} \kappa_{\ell}$ & $\kappa_{\mathrm{a}}^{2}$ & $\kappa_{\mathrm{a}}^{2} \kappa_{\ell}^{2}$ & $\kappa_{\mathrm{a}}^{3}$ & $\kappa_{\mathrm{a}}^{4}$ \\
FSL & 1 & $\kappa_{\mathrm{a}}$ & $\kappa_{\mathrm{a}}^{2}$ & $\kappa_{\mathrm{a}}^{2}$ & $\kappa_{\mathrm{a}}^{3}$ & $\kappa_{\mathrm{a}}^{4}$ \\
\hline
\end{tabular}

Table B.6: As in table B.4: male genotype fitness for strongly-suppressed non-homologous underdominance system. 


\begin{tabular}{lcccccc}
\hline \multicolumn{6}{c}{ Genotype } \\
Lethality & \multirow{2}{*}{$A A B B$} & $A \alpha B B$ & \multirow{2}{*}{$A \alpha B \beta$} & $\alpha \alpha B B$ & $\alpha \alpha B \beta$ & \\
& $A A B \beta$ & & $A A \beta \beta$ & $A \alpha \beta \beta$ & $\alpha \alpha \beta \beta$ \\
\hline BSL/FSL & 1 & $\kappa_{\mathrm{a}} \kappa_{\ell}$ & $\kappa_{\mathrm{a}}^{2}$ & $\kappa_{\mathrm{a}}^{2} \kappa_{\ell}^{2}$ & $\kappa_{\mathrm{a}}^{3}$ & $\kappa_{\mathrm{a}}^{4}$ \\
\hline
\end{tabular}

Table B.7: As in table B.4: female genotype fitness for strongly-suppressed non-homologous underdominance system.

\begin{tabular}{lcccc|cccc}
\hline & \multicolumn{4}{c}{ Male } & \multicolumn{2}{c}{ Genotype } & \multicolumn{2}{c}{ Female } \\
Lethality & $A A$ & $A \alpha, A \beta$ & $\alpha \beta$ & $\alpha \alpha, \beta \beta$ & $A A$ & $A \alpha, A \beta$ & $\alpha \beta$ & $\alpha \alpha, \beta \beta$ \\
\hline BSL & 1 & $\kappa_{\mathrm{a}} \kappa_{\ell}$ & $\kappa_{\mathrm{a}}^{2}$ & $\kappa_{\mathrm{a}}^{2} \kappa_{\ell}^{2}$ & 1 & $\kappa_{\mathrm{a}} \kappa_{\ell}$ & \multirow{2}{*}{$\kappa_{\mathrm{a}}^{2}$} & \multirow{2}{*}{$\kappa_{\mathrm{a}}^{2} \kappa_{\ell}^{2}$} \\
\hline FSL & 1 & $\kappa_{\mathrm{a}}$ & $\kappa_{\mathrm{a}}^{2}$ & $\kappa_{\mathrm{a}}^{2}$ & & \\
\hline
\end{tabular}

Table B.8: Genotype fitness for the homologous engineered underdominance system for bi-sex lethality (BSL) and femalespecific lethality (FSL). Genotypes with the same fitness are grouped.

\section{Analytical investigation of underdominance thresholds}

We may recast the population model in terms of proportions: if $n_{i}=N_{i} / N$ where $N=\sum_{j}^{n_{g}} N_{j}$ where $n_{g}$ is the number of genotypes, then

$$
\frac{\mathrm{d} n_{i}}{\mathrm{~d} t}=\frac{1}{N} \frac{\mathrm{d} N_{i}}{\mathrm{~d} t}-n_{i} \frac{1}{N} \frac{\mathrm{d} N}{\mathrm{~d} t} .
$$

Using (C.1), our governing equations for the proportions of adults $\left(n_{i}\right)$ and larvae $\left(b_{i}\right)$ in each genotype are

$$
\begin{aligned}
& \frac{\mathrm{d} b_{i}}{\mathrm{~d} t}=\frac{\rho N_{f}}{B} \tilde{p}_{i} \phi_{i}^{E}-F_{i}(B) b_{i}-b_{i}\left[\frac{\rho N_{f}}{B} \sum_{j}^{n_{g}} \tilde{p}_{j} \phi_{j}^{E}-\sum_{j}^{n_{g}} F_{j}(B) b_{j}\right], \\
& \frac{\mathrm{d} n_{i}}{\mathrm{~d} t}=\frac{m B}{2 N_{f}} b_{i} \phi_{i}^{L}-\mu n_{i}-n_{i}\left[\frac{m B}{2 N_{f}} \sum_{j}^{n_{g}} b_{j} \phi_{j}^{L}-\mu\right],
\end{aligned}
$$

where we have used the fact that $\sum_{i} n_{i}=1$, and we have defined $F_{i}(B)=\log \left[1+\left(\nu_{i} B\right)^{\eta}\right]+m+\mu_{B}$.

We implicitly assume that all genotypes share the same ecological parameters except for the possibility of competing unequally for resources at the larval stage. The $\tilde{p}$ are the proportion of offspring falling into each genotype, and $N_{f}$ is the sum of all adult females. We are interested in thresholds of the system (C.2). These are internal system states where all viable genotypes $k$ have $n_{k} \neq 0$ and $b_{k} \neq 0$. A small perturbation around the state will trigger a drive due to underdominance in favour of the positively perturbed homozygote (to the detriment of the negatively perturbed homozygote). We define

$$
\Phi^{L}=\sum_{j}^{n_{g}} b_{j} \phi_{j}^{L}, \quad \Phi^{E}=\sum_{j}^{n_{g}} \tilde{p}_{j} \phi_{j}^{E}, \quad \Theta=\sum_{j}^{n_{g}} F_{j}(B) b_{j},
$$

and investigate the non-trivial equilibria of (C.2), which satisfy

$$
\begin{aligned}
& 0=\frac{\rho N_{f}}{B}\left(\tilde{p}_{i} \phi_{i}^{E}-b_{i} \Phi^{E}\right)+b_{i}\left(\Theta-F_{i}(B)\right), \\
& 0=b_{i} \phi_{i}^{L}-\Phi^{L} n_{i} .
\end{aligned}
$$




\section{C.1. Equal larval competition}

We first investigate the simple case in which all genotypes compete equally at the larval stage, $\nu_{i} \equiv \nu$ $\forall i$, hence $F_{i}(B) \equiv F(B)$ and

$$
\Theta=F(B) \sum_{i}^{n_{g}} b_{i}=F(B)
$$

since $\sum_{i}^{n_{g}} b_{i}=1$. Under this assumption, (C.4) collapse into one equation for the adult genotype frequencies,

$$
\tilde{p}_{i} \phi_{i}^{E} \phi_{i}^{L}-\Phi^{E} \Phi^{L} n_{i}=0,
$$

which displays symmetry between early-acting and late-acting fitness costs. Summing (C.6) over $i$ in either the early- or late-acting regime produces $\Phi=\sum_{i}^{n_{g}} \tilde{p}_{i} \phi_{i}$ where the relation holds if the costs $\phi$ act either before hatching or during pupation. Thus we find the dispersion relation

$$
n_{i}-\frac{\tilde{p} \phi_{i}}{\sum_{j}^{n_{g}} \tilde{p}_{j} \phi_{j}}=0,
$$

from which we immediately see that the equilibrium genotype frequencies under the assumption of equal larval competition decouple from the effects of density-dependent mortality. Further, if all genotypes share the same ecological parameters (maturation rate, mortality rate etc.) then these parameters drop out of the calculation for the underdominance threshold.

If we simplify further by considering the six-genotype homologous underdominance system and assuming, as Davis et al. [25] did, that only the wild type $A A$ and the engineered construct $\alpha \beta$ are viable, then we can deduce from (C.7) that

$$
n_{1}=\frac{1}{2} n_{2} \phi_{2},
$$

(where we have re-numbered such that $i=1$ is wild type and $i=2$ is the construct, and non-viable genotypes have been discarded) and using the normalisation condition $n_{1}+n_{2}=1$ we find that for $\phi_{2}=1, n_{1}=1 / 3$ and $n_{2}=2 / 3$. These are genotypic proportions rather than allelic proportions [as in, e.g., 76], but we may compare these results to those found by Davis et al. [25] (wherein purely genetic crosses in non-overlapping generations were considered) by converting their results: the threshold lies at the point where the alleles $A, \alpha$ and $\beta$ are equally common; each $\alpha$ (and similarly $\beta$ ) allele is converted into one $\alpha \beta$ gene, whereas two $A$ alleles are required to make a single $A A$ gene, thereby making the genotypic proportions $2 / 3$ for $\alpha \beta$ and $1 / 3$ for $A A$, matching our results.

\section{C.2. The effect of density dependence}

Now we relax the assumption that all genotypes compete equally during the larval stage. First, consider the case where the genetic constructs impart an early-acting fitness cost, such that $\phi_{i}^{L}=1 \forall i$ and $\Phi^{L}=1$. From (C.4b) we find $b_{i}=n_{i}$; substituting for $b_{i}$ in (C.4a) leads to a new dispersion relation for the genotypic proportions,

$$
n_{i}-\frac{\frac{\rho N_{f}}{B} \tilde{p} \phi_{i}^{E}}{\frac{\rho N_{f}}{B} \sum_{j}^{n_{g}} \tilde{p}_{j} \phi_{j}^{E}-\left(\sum_{j}^{n_{g}} F_{j}(B) n_{j}-F_{i}(B)\right)}=0 .
$$


$\mathbf{7 2 5}$

726

727

728

If we again simplify down to two viable genotypes, $A A$ and $\alpha \beta$, we can find closed-form expressions for the threshold values. Using $\tilde{p}_{1} \rightarrow n_{1}^{2}$ and $\tilde{p}_{2} \rightarrow \frac{1}{2} n_{2}^{2}$ in the two-genotype limit (from (A.1a) and (A.1d)), the dispersion relation (C.9) reduces to the linear relation

$$
n_{1}=\frac{1}{2} n_{2} \phi_{2}^{E}+\frac{B}{\rho N_{f}}\left(F_{1}(B)-F_{2}(B)\right),
$$

which, together with the normalisation condition $n_{1}+n_{2}=1$ is enough to fully determine the threshold genotypic proportions as

$$
\begin{aligned}
& n_{1}=\frac{1}{1+\frac{1}{2} \phi_{2}^{E}}\left(\frac{1}{2} \phi_{2}^{E}+\frac{B}{\rho N_{f}}\left(F_{1}(B)-F_{2}(B)\right)\right), \\
& n_{2}=\frac{1}{1+\frac{1}{2} \phi_{2}^{E}}\left(1-\frac{B}{\rho N_{f}}\left(F_{1}(B)-F_{2}(B)\right)\right) .
\end{aligned}
$$

Increasing $\nu_{2}$ relative to $\nu_{1}$ or decreasing $\phi_{2}^{E}$ below unity decreases the $n_{1}$ threshold value below the $1 / 3$ which a purely genetic analysis predicts [25], making a population replacement in favour of $n_{2}$ (the engineered homozygote $\alpha \beta$ ) more costly. Thus, where ecological parameters differ between genotypes the underdominance threshold shifts; ecological effects that affect every genotype equally do not play a role in determining the underdominance threshold. 